\title{
Ultrafast electronic spectroscopy for chemical analysis near liquid water interfaces: concepts and applications
}

\author{
O. Link • E. Lugovoy $\cdot$ K. Siefermann $\cdot$ Y. Liu • \\ M. Faubel • B. Abel
}

Received: 13 December 2008 / Accepted: 27 February 2009 / Published online: 1 April 2009

(c) Springer-Verlag 2009

\begin{abstract}
Electron spectroscopy for chemical analysis (ESCA) being conceptually a photoelectron spectroscopy is established as a chemically specific probe mostly for surface analysis. Liquid phase ESCA for volatile liquids has become possible through the development of the liquid microjet technique in vacuum enabling the measurement of liquid interface photoelectron emission at the high vapor pressure of volatile liquids. Recently we have been able to add the dimension of time to the liquid interface ESCA technique employing high-harmonics soft X-ray and UV/near IR femtosecond pulses in combination with liquid water micro beams in vacuum. The concepts as well as technical details are outlined and several characteristic applications are highlighted.
\end{abstract}

PACS 72.30.+q $\cdot 73.20 .-\mathrm{r} \cdot 73.20 . \mathrm{Mf} \cdot 78.30 . \mathrm{Cp} \cdot 78.47 . \mathrm{jc} \cdot$ 78.47.J-

\section{Introduction}

1.1 ESCA and photoelectron spectroscopy near liquid interfaces

X-ray Photoelectron Spectroscopy (XPS), also known as Electron Spectroscopy for Chemical Analysis (ESCA), is a powerful spectroscopic tool for the study of the physical and chemical properties of a material's surface [2]. Core electrons are emitted by the sample due to the well-known photoelectric effect. The emitted electrons have a kinetic energy equal to the difference of the X-ray photon energy and the binding energy of the electron as illustrated in Fig. 1, hence for a known photon energy the conversion from kinetic energies to binding energies is straightforward, thereby enabling element identification. ESCA is only able to analyze the top 20-50 $\AA$ of a sample, making it an extremely surface-sensitive technique. In addition to the element specificity, the technique can also provide information about an element's chemical environment or oxidation state.

Due to the technical and conceptual problems with (volatile) liquids in vacuum, liquid phase (high pressure) ESCA is much less well established [3-8] than XPS at solid state surfaces. Only after Faubel et al. developed the liquid beam technique in vacuum also volatile liquids like water could be investigated with photoelectron spectroscopy in vacuum [5-8]. Since then, the chemical shift in the static ESCA approach has also been a particularly powerful observable for probing electron densities and molecular orbital energies in different liquid molecular environments $[9,10]$.

\subsection{Time-dependent chemical shifts}

In order to discuss time-dependent chemical shifts in ultrafast experiments we will start from the classical chemical 

the principle of photoelectron spectroscopy (high-energy photon produces an electron with a kinetic energy determined by the difference of the photon energy and the binding energy of the electron). From the kinetic energy spectrum the binding energy spectrum is calculated (adapted from [1])
Fig. 1 Schematic illustration of

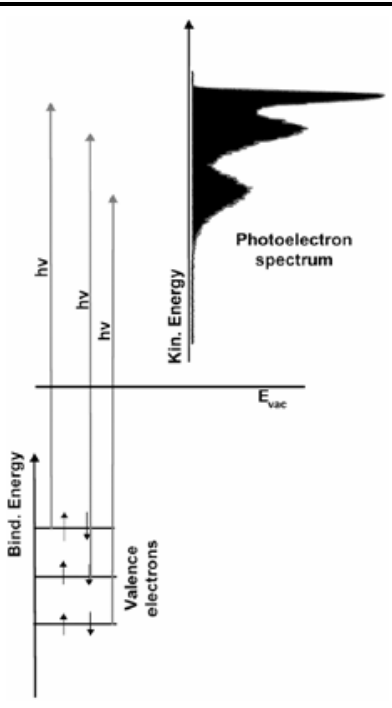

shift as introduced by Siegbahn et al. [2, 3]. As illustrated in Fig. 1 the detected kinetic energy spectra of the electrons in an ESCA or XPS experiment are converted to spectral emission intensities as a function of binding energy, thereby enabling element identification. The chemical environment of an atom affects the strength with which electrons are bound to it. Atoms associated with different chemical environments (intra- and intermolecular) produce photoemission peaks appearing at slightly different binding energies, which is referred to as the chemical shift. Binding energies and chemical shifts are used here interchangeably. The term "chemical shift" results from comparative studies in series of ESCA studies of atoms in molecules (e.g., C-atoms) with different chemical bonding or with different chemical substitution. It is in fact correlated with electron densities in the molecules, as has been acknowledged early by theoreticians [2-4], and it has early been pointed out that there exists a correlation between chemical shifts in NMR and ESCA both correlated with electron densities in the molecule [11]. For a full and comprehensive theoretical account of the chemical shift we refer to [11].

Taking into account the initial and final states of relevance (see also Fig. 2) and energy conservation the electron binding energy is

$E_{B}=E_{\mathrm{tot}}^{f, \mathrm{HF}}-E_{\mathrm{tot}}^{i, \mathrm{HF}}+\Delta E^{\mathrm{corr}}+\Delta E^{\mathrm{rel}}$,

where $E_{\text {tot }}^{i, \mathrm{HF}}$ denotes Hartree-Fock energies of the final and initial states, respectively, and $\Delta E^{\text {corr }}$ and $\Delta E^{\text {rel }}$ is the differences of the correlation and relativistic energies of the final and initial states [11]. The reference level in electron binding calculations is generally chosen as the vacuum level. Following Koopman's theorem, which says that in the frozen orbital approximation the electron binding energy is equal to the negative of the Hartree-Fock orbital energy of

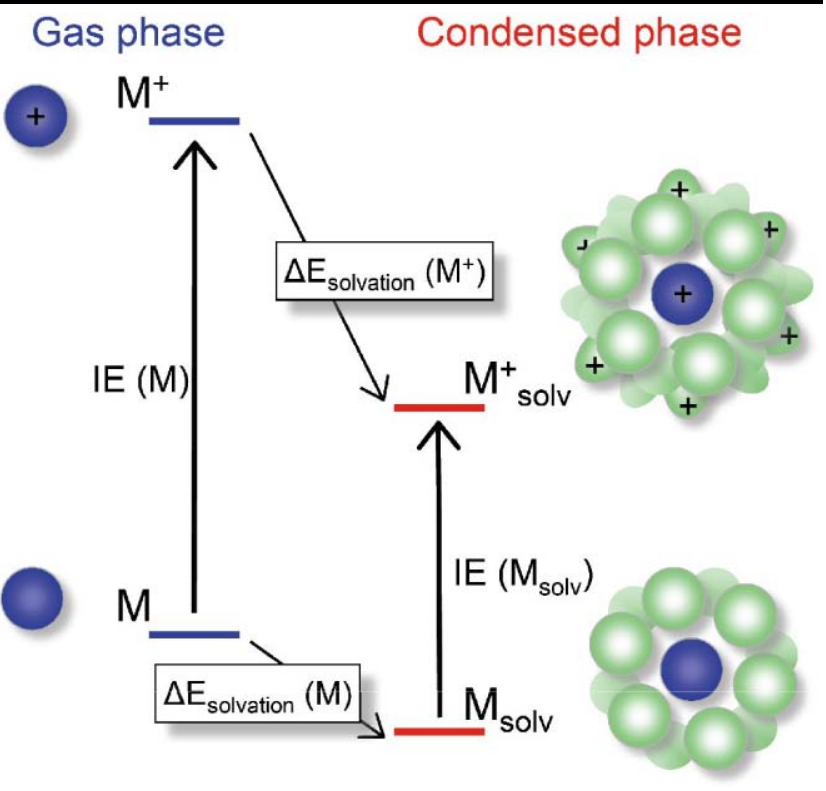

Fig. 2 Scheme illustrating the features of photoelectron spectroscopy (ESCA or XPS) and the chemical shift in different molecular environments. The ionization of an atomic system in vacuum and in a dense environment is shown in both cases (left and right). In the dense environment the polarization of the medium is also displayed. The lengths of the vertical arrows reflect photon energies and chemical shifts. Here the desolvated and the solvated system are shown schematically. It is obvious that the photoemission and in turn the chemical shift depend strongly upon the environment. For neutrals the effect of polarization and the features of the ionized cationic state are at least as important as the orbital energy of the neutral species. Time-dependent ESCA (monitoring for example the desolvation of the neutral atomic system in this simple case) traces all the illustrated processes in a relatively complex time-dependent manner. In static ESCA or XPS this is usually not a problem

the ground state, $-\varepsilon^{\mathrm{HF}}$, we may write [11]

$E_{B}=-\varepsilon^{\mathrm{HF}}+E^{\mathrm{reorg}}+\Delta E^{\mathrm{corr}}+\Delta E^{\mathrm{rel}}$,

where $E^{\text {reorg }}$ is the reorganization energy, also called relaxation energy.

What appears to be a non-trivial but straightforward problem in the static ESCA approach is much more complicated for the time-dependent case. Since the initial and final states and all processes affecting them are time dependent, the time-dependent chemical shifts are governed by the complicated interplay of all states and processes involved. In Fig. 2 such a situation is depicted for a simple solvation/desolvation of an atomic system. In order to avoid confusion we note that the time-dependent chemical shift here is related to photoelectron emission intensities at specific binding energies, keeping consistency with the original term introduced by Siegbahn. Any time-dependent evolution of photoemission lines, which is characterized for example by the evolution of the line maximum in time should not be termed a time-dependent chemical shift. Instead, its timedependent projection onto the binding energy axis results 
in the time-dependent chemical shifts or time-dependent binding energies. Any binding energy and chemical shift (i.e., a point on the $x$-axis) in time-dependent spectra carries in general a number of contributions from intramolecular electron density and from the distribution of molecules with slightly different intermolecular interactions and environments. These contributions are not static like in classical ESCA, but time-dependent, making the traditional "inversion" and assignment of spectra much more demanding. In Fig. 2 we have plotted a simple graph which is commonly used to evaluate electron binding energies. Let us assume a time-dependent laser induced evaporation process that transfers the atom from the condensed liquid phase into the gas phase on a fast time scale. (Such an experiment is in fact described in Sect. 3.2.) During the process (from right to left) the photoemission line of the system changes in time. This is clearly a result of the changed environment of the neutral and the ionized state. The energies of both depend differently on the changes in the molecular environment (density) in time, which would result in a timedependent shift of the photoemission line towards higher energies. In order to avoid confusion we do not consider the shift of bands as chemical shifts here. Instead, their evolution in time can in turn be analyzed by the changing intensity at specific binding energies corresponding to chemical shifts, which carry photoemission intensity from a number of (overlapping) species and contributions. Alternatively, we may consider a time-dependent process such as a typical chemical reaction in the condensed phase. Also, the photoemission signal and line intensities depend upon the upper ionic and lower neutral state and its time dependence. The projection onto the binding energy axis provides chemical shifts as a function of time. Cuts through the spectra at a single energy display the chemical shift or the binding energy as a function of time. If theory is able to map out electronic energies (in time) for the ground state and the ionic state it should be possible map out the electron orbitals (electron densities) of the molecules in a timeresolved ESCA experiment by solving the "inversion" problem.

Fig. 3 Classical

three-step-model [14] for high-harmonics generation. (a) Electron tunneling in strong laser fields. (b) Acceleration, deceleration and recollision of electrons with the core in intense laser fields. (c) Emission of a high-energy photon after recollision. For more details see the text
The long-time goal of the present approach is therefore to extend ultrafast ESCA to core and valence electrons (participating in chemical bonding) and to probe and trace electron orbital energies and thus "orbitals" in time in chemical reactions or other relevant processes in biology and physics. We anticipate that it may be possible to classify chemical reactions by their orbital autocorrelation functions, which may be probed by experiments conceptually highlighted in the present contribution. The realization of this approach (theory and experiment) is in progress in our group at present.

\subsection{High-harmonic generation}

High Harmonic Generation (HHG) was observed first in the end of the 1980s [12]. The high-harmonic spectra were found to decrease in intensity at low orders but then form a plateau, whereby the intensity of the harmonics remain approximately constant over many orders [12]. Plateau harmonics spanning hundreds of electron volts have been measured which extend into the soft X-ray regime [13]. This plateau ends abruptly at a position called the high-harmonic cut-off.

Radiation provided by high-harmonic generation results from the addition of fields emitted by individual atoms, among which the propagation of the driving laser beam introduces phase relations. The widely accepted classical picture of high-harmonics generation has been provided by Corkum et al. some time ago [14]. The main features and steps are given in Fig. 3.

Coherence is an original property of high harmonics. The phase transfer from the laser to the emitting atoms occurs via a medium of space-time varying refraction index, where the phase matching conditions strongly depend on the laser intensity. Therefore, phase matching optimization has a great part in the ultimate harmonic properties, especially coherence, angular distribution, intensity, and energy cut-off [15].

For the position of the cut-off at the plateau end, it is determined by the atom ionization potential, $I_{p}$, and by the ad- a

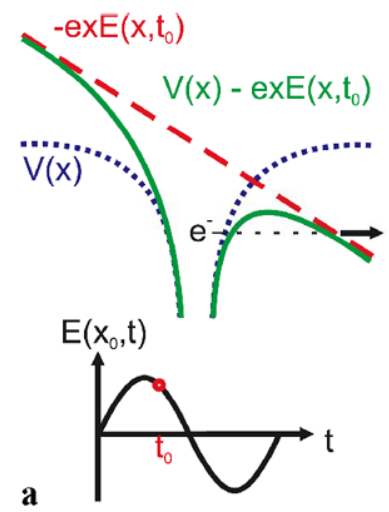

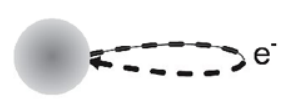

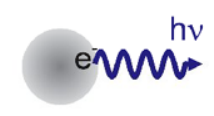

b

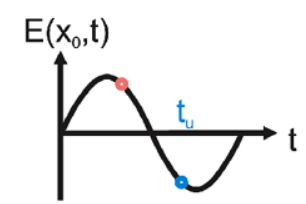

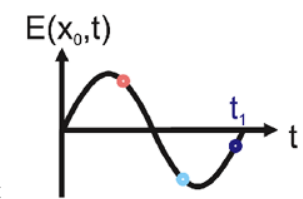


ditional ponderomotive potential, $U_{p} . U_{p}$ has the form [14]

$U_{p}=\frac{e^{2} E^{2}}{4 m \omega^{2}}$

for a linearly polarized oscillating field, $E \sin \omega t$. Replacing $E$ and $\omega$ by the laser intensity, $I_{L}$, and wavelength, $\lambda$, (3) becomes

$U_{p} \cong 9.33 \times 10^{-14} I_{L} \lambda^{2}$.

Now the initial velocity of an ionized electron varies, with the same frequency $\omega$ as above, as a function of the time $t_{0}$ at which this electron has been released in the electric field. Therefore, the time-averaged kinetic energy of electrons must be a function of the phase $\omega t_{0}$ of the field at time $t_{0}$. This energy is calculated to be [16]

$\left\langle\frac{1}{2} m v^{2}\right\rangle=\frac{e^{2} E^{2}}{4 m \omega^{2}}\left(1+2 \cos ^{2} \omega t_{0}\right)$

from which one recognizes that the energy gained by electrons oscillating in the field should be distributed between $U_{p}$ and $3 U_{p}$. Consequently the maximum energy of harmonic photons is expected near a value $E_{\max }$ given by

$E_{\max }=I_{p}+\alpha U_{p}$,

where $\alpha=3$ in the present classical model. Quantum calculations confirm the linear scaling of the cut-off energy with both ionization potential and laser intensity and provide a better accuracy of the value of $\alpha$. Single atom calculations $[14,17]$ lead to $\alpha=3.17$ which agrees with the large number of experimental observations. With helium it has been shown that it is possible to generate high harmonics even beyond $1 \mathrm{keV}$ [13].

Although there are several approaches and experimental realizations to provide the medium for high-harmonics generation, namely, the jet technique [18], the capillary technique $[19,20]$, the drilled capillary technique [1] or even HHG near nanostructures [21], we have strongly favored the drilled capillary technique [1], because it provides high conversion efficiencies and stability. The present experiment also makes use of the advantage of having a very collimated EUV laser-like pulse that can easily be focused.

Intense tunable EUV or XUV radiation sources are desirable for many applications. In the case of generation and separation the distribution of many harmonics (if one is selected) enables a coarse tuning of the radiation source. The separation of one harmonics (here with a grating) is a prerequisite for the present experiment. If time resolution is important, a pair of gratings or multilayer mirrors has to be used. If the wavelength has to be tuned in more detail, the fundamental of a Ti:Sapphire system may be tuned as well. For photoelectron spectroscopy and XPS this is not necessary. High harmonics are also the basis of attosecond pulses and thus of the field of attosecond physics [19, 22-32].
1.4 Time-resolved photoelectron spectroscopy near liquid interfaces

The combination of several powerful technologies, i.e., photoelectron spectroscopy near volatile liquid interfaces in vacuum [10], ultrafast pump-probe spectroscopy, and tabletop high-harmonic generation of soft X-ray radiation [33] enabled us to add the dimension of time to the liquid interface ESCA or XPS technique [1]. Our first experimental setup is described in [1]. Recently, Drescher has highlighted a similar concept for gas phase processes occurring primarily on attosecond and low-femtosecond time scales $[32,34]$.

Conceptually, time-resolved liquid phase photoelectron spectroscopy relies first and foremost on a vacuum incompatible liquid (water or other volatile solvents) interface in vacuum. We use a fast flowing high-pressure liquid microjet in vacuum. The temperature as well as thermodynamic properties of the beam in vacuum can be characterized well, as has been shown by us and others [5]. The pumping conditions and the vacuum have to be arranged in such a way that the mean free path for photoelectrons in collisions with surrounding solvent molecules in the vicinity of the beam is minimized [7]. For the EUV spectral range $(50-100 \mathrm{eV})$ the escape depth $z$ of photoelectrons from the interface of the liquid beam is on the order of a few monolayers [35]. Only photoelectrons generated in these first layers of the liquid surface can escape and (with a negligible atmosphere around the micro beam avoiding inelastic collisions) be detected. In order to measure the photoelectron kinetic energies the electrons have to be sampled with an adjustable $100 \mu \mathrm{m}$ micro skimmer very close to the liquid beam surface. Since the current approach is photoelectron spectroscopy at relatively high pressures, the skimmer at the spectrometer entrance has to be differentially pumped.

For a time-resolved experiment an ultrafast EUV pulse has to be available, preferentially in a table-top arrangement. In the past, photoelectron experiments near liquid interfaces have been employing quasi-continuous synchrotron radiation, only. The present experiment is the first one in which a high intensity short (high-harmonic) pulse is used for liquid phase PES. Initially it was not clear at all whether such an experiment could be successful because of transient space charges and long-time charging of the beam in general, which may cause severe problems for photoelectron spectroscopy near liquid interfaces. These problems have been solved by carefully adjusting the photon intensities and by employing conducting gold layers covers at the tip of the nozzle.

One of the highly collimated laser-beam-like high harmonics of the $800 \mathrm{~nm}$ fundamental in vacuum is selected with a EUV grating and focused with a toroidal mirror on 
Fig. 4 Left panel: The pump and probe beam arrangement and overlap on the water beam in front of the micro skimmer. Right panel: The quartz micro nozzle producing a small water filament of $10-20 \mu \mathrm{m}$ diameters. The micro water beam displayed in front of the spectrometer entrance where a skimmer of $100 \mu \mathrm{m}$ diameter is mounted

Fig. 5 Scheme of the optical setup (adapted from [1]), providing the IR/UV-pump pulse $(2.6-3.0 \mu \mathrm{m} / 270 \mathrm{~nm})$ and the EUV-probe pulse (e.g., at $39 \mathrm{eV}$ )
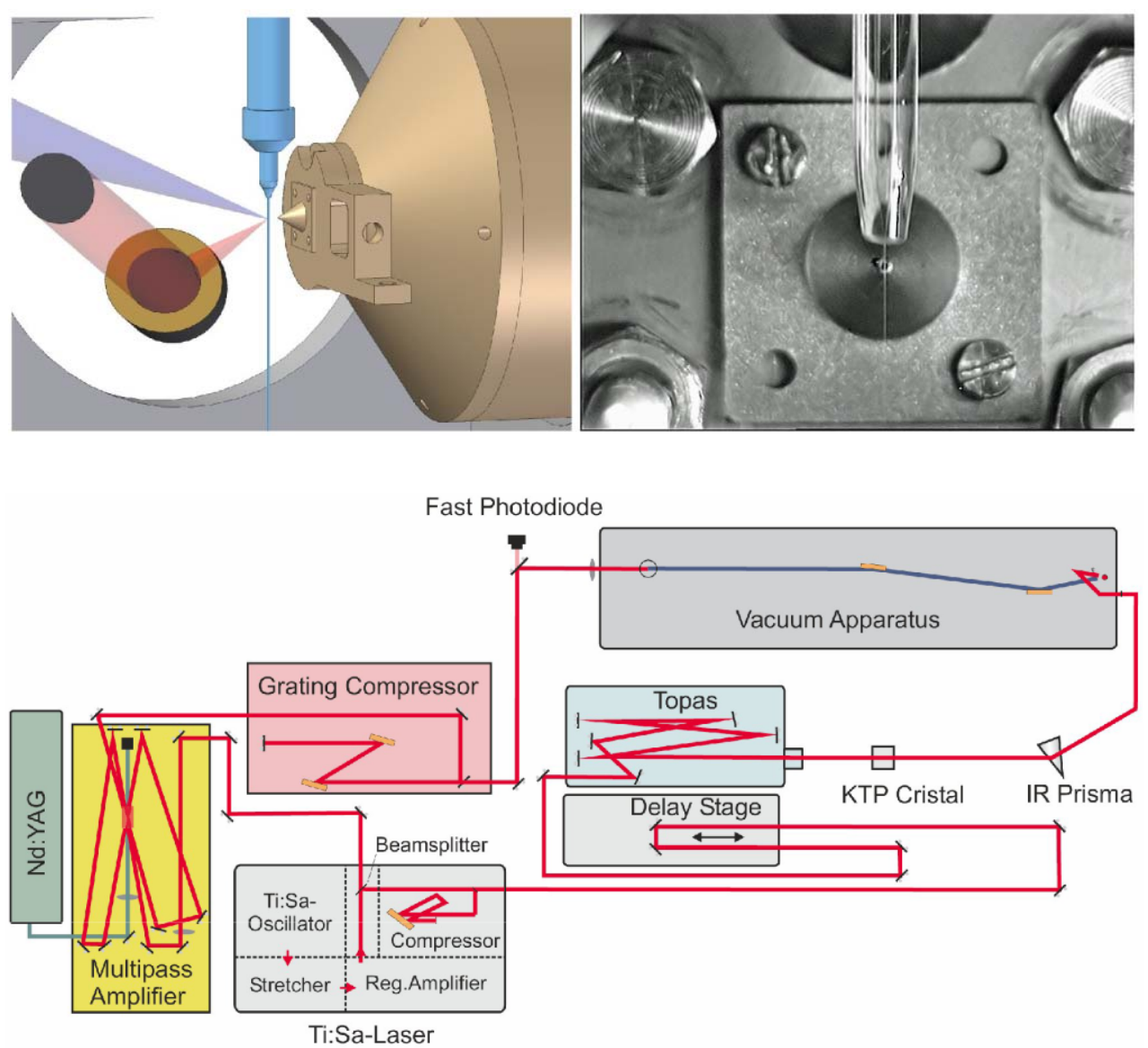

the liquid jet (measured focus diameter $\approx 100 \mu \mathrm{m}$ ). For the ultrafast liquid interface ESCA technique to work, the excitation pulse and the EUV (high-harmonics) probe beam have to be overlapped nearly collinearly in space and time on the microjet (Fig. 4). The liquid microjet and the pump and probe beam geometry in front of the time-of-flight electron spectrometer in vacuum is displayed schematically for an IR and an EUV pulse pair in Fig. 4.

The time-of-flight electron spectrometer used in the experiments enables us to record the entire photoelectron spectra (multiplex advantage) for predefined time-steps of the delay of the two laser pulses. Shot-to-shot-variations and intensity drifts of the high-harmonic radiation could be avoided through averaging, discrimination, and normalization with reference spectra at negative delays for each time point. The time-of-flight data are finally converted into kinetic energies of the photoelectrons and in turn into binding energies. With this technology time-dependent photoelectron spectra as a function of delay time between the pump and the EUV probe pulses have been recorded. From the spectra read out at specific positions kinetic traces for one particular chemical shift or binding energy are obtained [1].

\section{Experimental}

\subsection{Amplified femtosecond laser system and setup}

In this experiment a commercial Ti:Sapphire laser system (Hurricane, Spectra Physics) delivers short pulses with a length of $100 \mathrm{fs}$ and a energy of $1.2 \mathrm{~mJ}$ at a repetition rate of $1 \mathrm{kHz}$. The laser system employs the chirped pulse amplification (CPA) technique which incorporates typically four components, namely a Ti:Sa oscillator, a grating stretcher, a regenerative amplifier, and a grating compressor (Fig. 5).

The internal laser setup was slightly modified by exchanging a mirror with a beam splitter in front of the internal compressor. Three thirds of the internally amplified radiation were reflected by the beam splitter and temporally compressed by the internal compressor to give $780 \mathrm{~mW}$ of the standard $100 \mathrm{fs}$ Hurricane output. The remaining $380 \mathrm{~mW}$ of the amplified but uncompressed pulse were transmitted and coupled out towards an external multi-pass amplifier. There an amplification up to $2 \mathrm{~W}$ takes place by three passes through an additional Ti:Sapphire crystal (pumped by $10 \mathrm{~W}$ of a Nd:YAG laser). Subsequent compression in an external grating compres- 
sor led to pulses of $1.5 \mathrm{~mJ}$ energy at $100 \mathrm{fs}$ pulse duration.

\subsection{IR radiation at $2.6-3.0 \mu \mathrm{m}$}

The $780 \mathrm{~mW}$ of the regular Hurricane output were sent to an optical parametric amplifier (TOPAS, Light Conversion) for photon conversion into the infrared (Fig. 5). Although the standard model of the TOPAS only allows for the generation of near IR radiation down to $2.5 \mu \mathrm{m}$ due to the nonlinear materials employed, an extension of the IR wavelength into the $3 \mu \mathrm{m}$ region was possible using the TOPAS output, which was mixed with the remaining fundamental in a second nonlinear process. In a subsequent KTP crystal placed $15 \mathrm{~cm}$ behind the TOPAS exit, an additional wave-mixing process of the generated IR around $1.1 \mu \mathrm{m}$ and the rest of the fundamental radiation at $800 \mathrm{~nm}$ took place [36]. Difference frequency generation and parametric amplification lead to radiation in the desired wavelength region at $2.6-3.0 \mathrm{~mm}$. By optimizing the TOPAS settings, pulse energies in excess of $10 \mu \mathrm{J}$ in the whole region could be obtained (even higher energies of $>30 \mu \mathrm{J}$ at $2.7 \mu \mathrm{m}$ ). Finally, an INFRASIL quartz prism was used to separate the IR radiation from the rest of the fundamental radiation and from other wavelength coming from the different wave-mixing processes.

The IR-pump pulse is coupled into the experimental chamber (see Fig. 6) via a $\mathrm{CaF}_{2}$-window and focused onto the liquid filament with a $60^{\circ}$-off-axis parabolic mirror (Fig. 6). The mirror has an aluminum surface, a focal length of $34 \mathrm{~mm}$ and leads according to knife edge measurements to a focus diameter of 70-100 $\mu \mathrm{m}$ (somewhat larger than the filament's diameter).

\subsection{High-harmonic generation}

The externally amplified $1.5 \mathrm{~mJ} 800 \mathrm{~nm}$ fundamental pulses from the multi-pass amplifier were employed for the generation of high harmonics. The required peak power of $>10^{14} \mathrm{~W} / \mathrm{cm}^{2}$ is obtained by focusing the short pulses with a $150 \mathrm{~mm}$ lens into an argon-filled capillary of $2.3 \mathrm{~mm}$ inner diameter, resulting in $4-8 \times 10^{14} \mathrm{~W} / \mathrm{cm}^{2}$. The argon pressure inside the capillary was typically about 50-200 mbar. The radiation enters and leaves the capillary through laserdrilled holes, which have a lifetime-dependent diameter of 100-300 $\mu \mathrm{m}$. In order to optimize the high-harmonic output the phase mismatch between the fundamental and the harmonic radiation in the interaction region must be minimized. This can be achieved by adapting the argon pressure and by tuning the parameters of the incident light through an iris in front of the focusing lens.

The vacuum chamber is pumped by a roots pump $\left(350 \mathrm{~m}^{3} / \mathrm{h}\right)$ to remove the leaking argon from the chamber and keeping the background pressure at a level of $10^{-2} \mathrm{mbar}$ in order to minimize the re-absorption of the XUV radiation which is under the present conditions less than $10 \%$ [15].

In the high-harmonic generation process several harmonics of the fundamental radiation are generated (Fig. 7) such that a dispersive element is necessary to separate a particular harmonic frequency. Therefore a spherical diffraction grating (700 lines $/ \mathrm{mm}$ ) is illuminated at a deviation angle of $161.8^{\circ}$ exploiting the total external reflection (Fig. 6). The grating has a radius of $17100 \mathrm{~mm}$ and is therefore not focusing but collimating the high-harmonic pulse.

To protect the grating from thermal damage by the fundamental radiation, a $150 \mathrm{~nm}$ aluminum filter can be intro-
Fig. 6 Schematic drawing of the vacuum apparatus consisting of four chambers and the TOF photoelectron spectrometer. In the inset the overlap of the IR-pump and the extreme ultraviolet or soft X-ray (EUV or XUV) probe pulse on the water filament is depicted

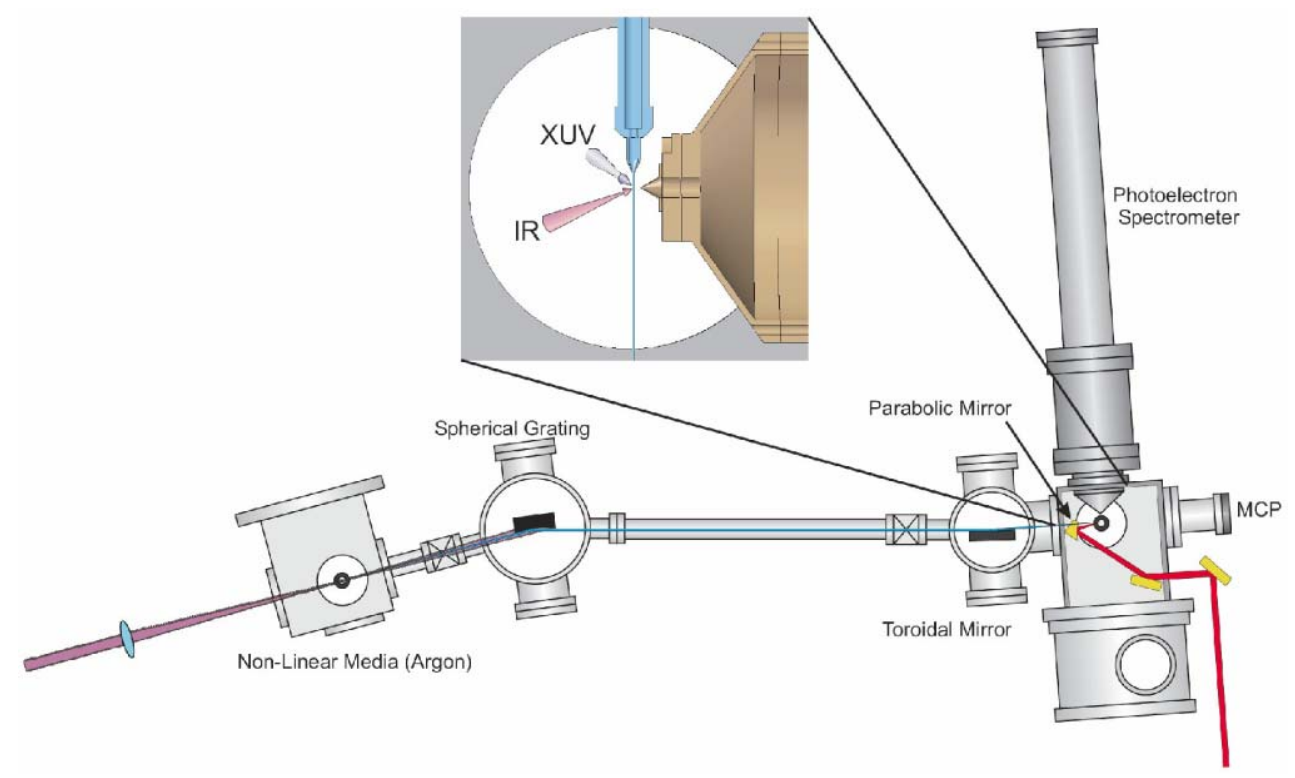


Fig. 7 Generation of high harmonics of the fundamental $800 \mathrm{~nm}$ radiation in a neon- or argon-filled capillary.

(a) A sequence of high harmonics generated in neon, imaged by a XUV-sensitive CCD-camera. The range of photon energies spans $40-70 \mathrm{eV}$. (b) A sequence of high harmonics generated in argon.

The range of photon energies in this case is between 25 and $40 \mathrm{eV}$. Note: it is only possible to generate the odd harmonics of the fundamental in both cases

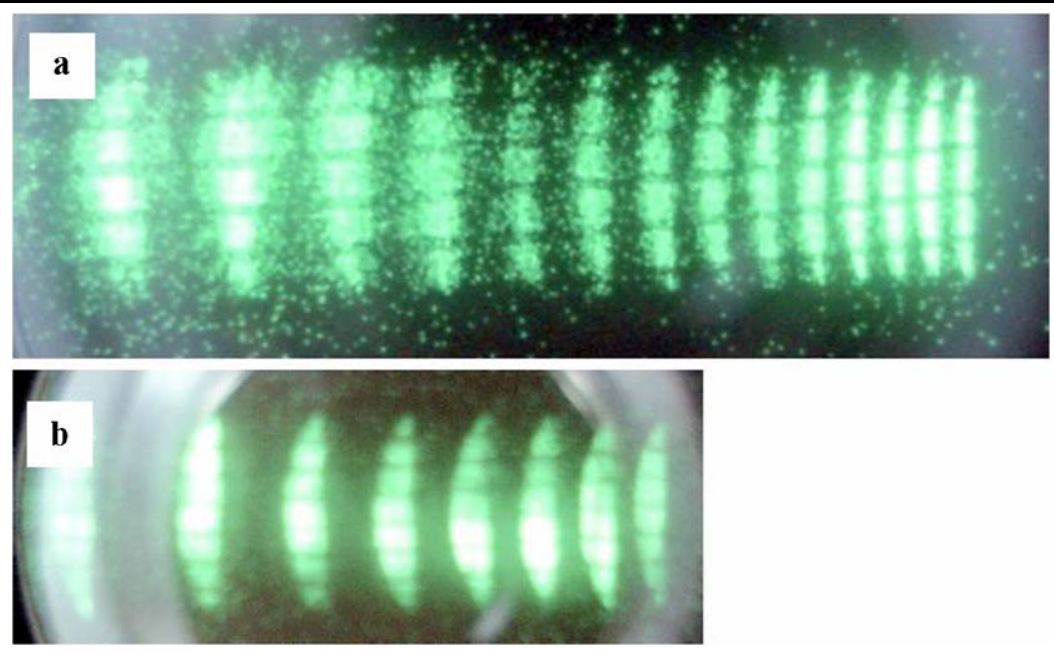

duced in the optical path in front of the grating. In the experiments reported here the 25 th harmonic of the $800 \mathrm{~nm}$ radiation was used, corresponding to a wavelength of $32.1 \mathrm{~nm}$ and to photon energy of $38.6 \mathrm{eV}$.

The nearly collimated XUV-probe beam is irradiated under grazing incidence onto a toroidal mirror which has an imaging length of $2 f=360 \mathrm{~mm}$ [37]. The mirror is covered with gold, has a size of $20 \times 100 \mathrm{~mm}$ and radii of curvature of 24.1 and $5375.3 \mathrm{~mm}$. The focus dimensions and quality depend strongly on the irradiation parameters, namely the incident angle and the deviation. The focal point is asymmetric but nevertheless a diameter in the spectrometer plane of $\approx 100 \mu \mathrm{m}$ can be achieved. The XUV radiation can be monitored with a XUVsensitive CCD camera (OD2562Z, Proxitronic), which is installed on the optical axis on the backside of the apparatus (Figs. 6, 7). Thus it is possible to see the liquid jet shadow in the XUV-image, which is useful for alignment purposes.

\subsection{Vacuum chamber and time-of-flight photoelectron spectrometer}

In the experimental chamber the IR-pump and the XUVprobe beam are overlapped in space and time on the liquid target (Fig. 6). The target is a liquid microjet, which was introduced by Faubel [5-8] some time ago (Fig. 4). A flux of $0.3-0.4 \mathrm{ml} / \mathrm{min}$ of the solvent is pressed through a micro nozzle with a HPLC-pump (Economy 2/ED, Microliquids $\mathrm{GmbH}$ ). The nozzle diameter is in the range of 10 $20 \mu \mathrm{m}$ giving a liquid filament in the experimental chamber of more or less the same diameter (Fig. 4). At velocities of $10-100 \mathrm{~m} / \mathrm{s}$ and with water the filament remains continuous for about 2-3 mm until it breaks up into a stream of droplets, which is frozen out in a cooling trap with liquid nitrogen. A second cooling trap inside the chamber acts as a strong pump for gaseous water and allows in combination with a turbo pump $(1600 \mathrm{l} / \mathrm{s})$ to keep the background pressure in the working apparatus at a level of $2 \times 10^{-4} \mathrm{mbar}$.

For detection of the emitted photoelectrons from the liquid water surface a time-of-flight electron spectrometer (Kaesdorf) is mounted to the side of the experimental chamber (Fig. 6). A skimmer of $100 \mu \mathrm{m}$ diameter separates the experimental chamber from the spectrometer and forms a differential pumping stage towards a second inner entrance of $2 \mathrm{~mm}$ diameter. Thereby a final vacuum better than $10^{-7}$ mbar can be maintained in the spectrometer under operation. The photoelectrons ejected from the liquid filament are collected in a narrow solid angle. Field free conditions are achieved by a 3D Helmholtz coil construction to compensate for the earth magnetic field and other disturbing magnetic fields. Under these conditions some of the photoelectrons enter the several hundreds micrometer distant skimmer and continue over a $60 \mathrm{~cm}$ long free flight distance until they are finally accelerated $(6.9 \mathrm{~cm}, 280 \mathrm{~V})$ for higher detection probability towards a MCP-detector. The $40 \mathrm{~mm}$ diameter MCP cuts out a solid angle of $3.5 \mathrm{msr}(0.028 \%$ of a $4 \pi$ sphere) of a $3 \mathrm{D}$ isotropic electron emission.

Figure 8 shows a photoelectron spectrum of gaseous water (with a little contribution of liquid water) taken from the surrounding gas phase at the liquid microjet. From the spectra we determined an energy resolution of about $0.25 \mathrm{eV}$, which was not optimized in the present experiments (specified resolution is close to $0.1 \mathrm{eV}$ ).

\subsection{Time resolution and autocorrelation function}

After a static photoelectron signal through the XUV-probe pulse is achieved the overlap with the IR-pump pulse must be realized. Therefore, the ability of the IR to generate plasma on the liquid filament is used to simplify the 2D adjustment towards a 1D adjustment, the filament acting as an inverse slit-like pinhole. 


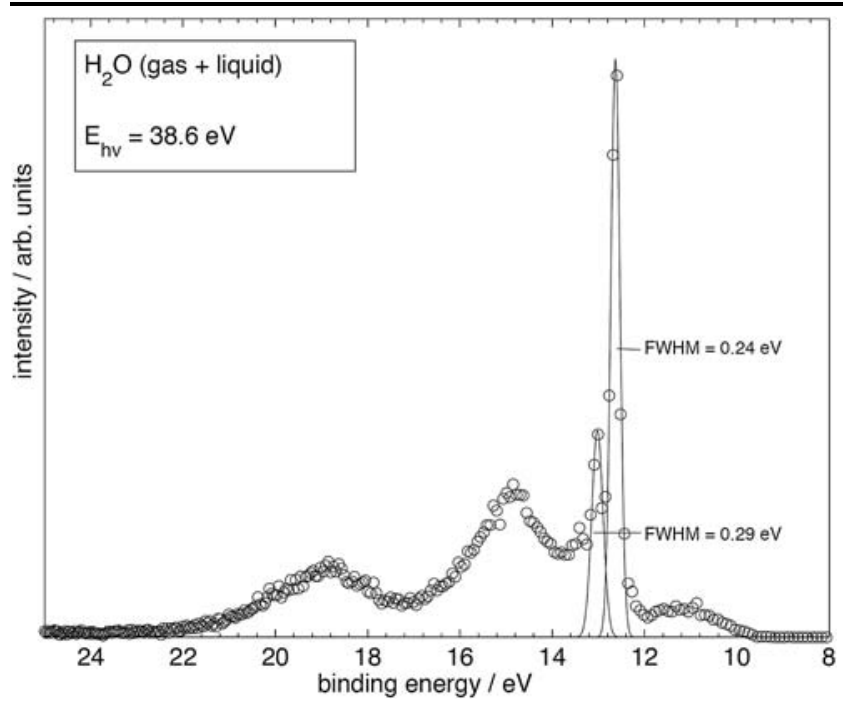

Fig. 8 A photoelectron spectrum of gaseous and some liquid water recorded near the liquid water beam. The spectrum shows the energy resolution of the spectrometer to be around $0.24 \mathrm{eV}$

Provided that the pulses are temporally overlapped, the spatial overlap can be detected (in the photoelectron spectrum) as the appearance of electrons additionally accelerated in a mixed IR-XUV-multiphoton process. This effect (signal) can also be utilized to obtain a cross-correlation to estimate the pulse length of the XUV-probe pulse (Fig. 9). Evaluating this process, which is highly nonlinear for the IR radiation an upper boundary for the XUV-pulse can be estimated to be 527 fs (the pulse length of the IR is known to be around 150-200 fs [36]). In the actual setup such an elongated EUV pulse is unavoidable as only one diffraction grating without recompression of the pulse is used. In an upgrade of the experiment in Göttingen, an improvement of the temporal resolution by using a second recompressing grating [18] or by exchanging the grating through multilayer mirrors $[34,38]$ is underway at present in our laboratory.

\section{Application results and discussion}

After the detailed experimental section we will highlight a number of characteristic applications demonstrating the potential of the technique and the general approach. Besides some time-resolved applications we have also included two spectroscopic experiments in which the high-harmonics radiation has been used only. Since high harmonics have not been used for photoelectron spectroscopy near liquid interfaces before, we want to show here by illustrative and wellknown examples that such a setup may replace to some extent synchrotron beam line sources in the future due to sufficient photon numbers in the EUV and a good sensitivity and dynamic range.
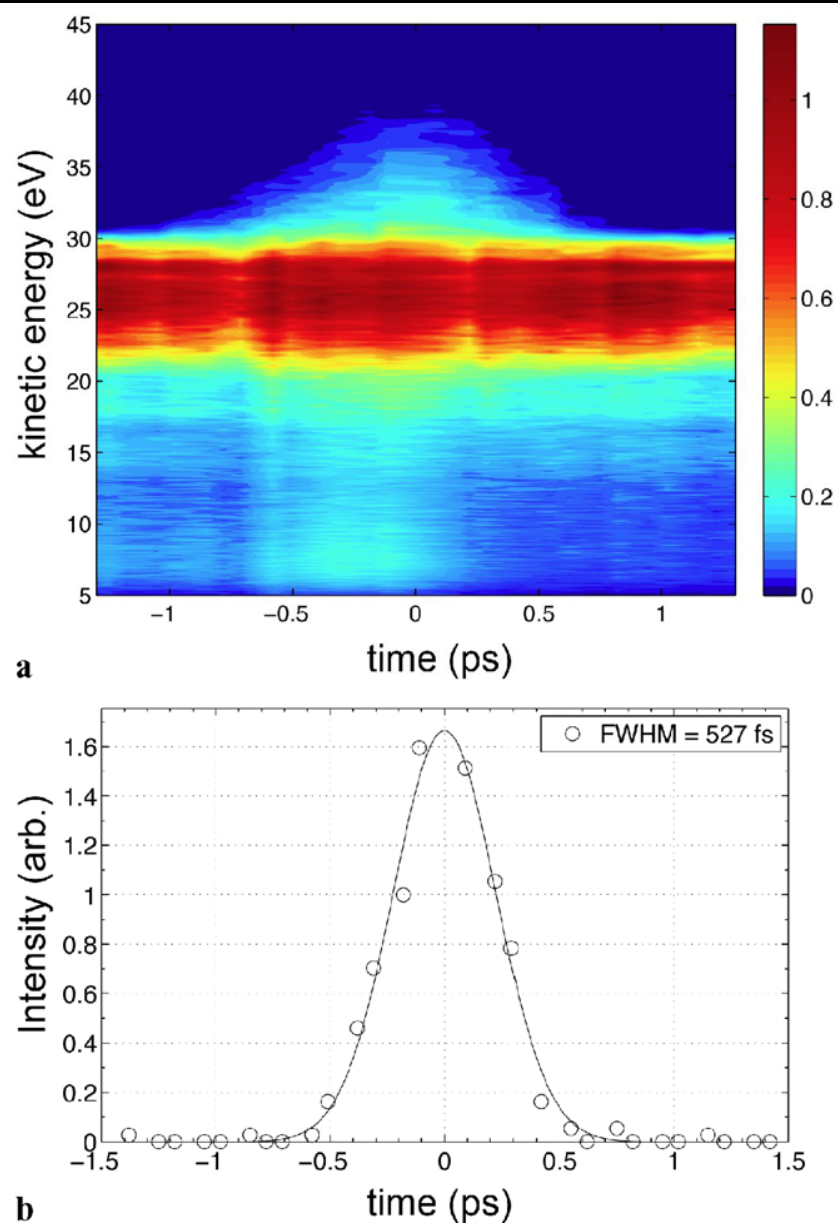

Fig. 9 (a) Kinetic energy plot of photoelectrons vs. time. If both laser pulses are overlapped in space and time on the liquid beam in front of the skimmer of the time-of-flight electron spectrometer, the EUV pulse detects fast electrons on top of the water photoelectron spectrum resulting both from the IR excitation and the EUV probe on the target. (b) A cut through the 2D-plot displayed in (a) providing an approximate cross-correlation function of the IR-pump and the XUV-probe pulse. Since the IR-pump pulse is significantly shorter the width of the cross-correlation is close to the width of the EUV pulse (upper boundary), broadened by the diffraction grating

\section{1 'Static' liquid XPS spectra by a table-top EUV high-harmonic radiation source}

\subsubsection{Chemical and surface sensitivity and selectivity}

The ultrafast table-top "beam line" was used to record what we call "static photoelectron spectra" of aqueous salt solutions, namely of $\mathrm{K}_{4}\left[\mathrm{Fe}(\mathrm{CN})_{6}\right]$ and tetrabutyl ammonium iodide (TBAI), without prior pump pulse excitation. Spectra of aqueous $\mathrm{K}_{4}\left[\mathrm{Fe}(\mathrm{CN})_{6}\right]$ have already been presented by Winter et al. [10, 39], who investigated similar solutions with synchrotron radiation at their liquid beam setup installed at the BESSY in Berlin. The very first investigations of a TBAI-solution had even been carried out in 1984 by Ballard with $\mathrm{He}(\mathrm{I})$ radiation [40]. At this point we show that also 
the Göttingen high-order harmonic radiation setup is capable of monitoring dissolved salt ions in an aqueous solution, which has so far mainly been demonstrated by the more intense synchrotron radiation.

ESCA and XPS as well as our time-resolved variant are surface-sensitive techniques as they monitor only those photoelectrons from layers near the surface of a liquid sample according to their very small escape depth of few nanometers or less. As for measurements of neat solvents this potentially allows for the investigation of molecules at the surfaces with some discrimination of bulk molecules from deeper layers, being able to monitoring even special surface states [41, 42]. In terms of the investigation of dissolved samples this surface sensitivity creates very special challenges and possibilities, namely only species with a significant surface concentration can be probed. In order to increase the concentration above the detection limit one can either provide a sufficiently large global concentration ( $\sim 3$ molar as for alkali-halides [10]) or investigate surfaceactive substances which accumulate from bulk forming higher local concentrations on surfaces.

An example for an interesting surface-active system that rapidly accumulates at the liquid water surface and in turn assembles in monolayers, also used as a phase transfer catalyst, is tetrabutyl ammonium iodide (TBAI). The large hydrophobic But $\mathrm{N}^{+}$cation exhibits only a small charge density and is therefore driven to the hydrophobic surface of the liquid water beam. According to measurements performed by Winter et al. a complete monolayer of dissolved salt covers the water surface, if the global concentration exceeds $0.02 \mathrm{~mol} / \mathrm{kg}$. Interestingly, the negatively charged iodide anions follow the positive cations and can be detected in significantly increased concentrations near the liquid interface as well, as shown in Fig. 10.

In Fig. 11 a spectrum of an aqueous solution of potassium ferrocyanide is displayed. In order to detect the iron and potassium ions for his particular case a fairly high concentration of $0.5 \mathrm{~mol} / \mathrm{l}$ has to be used. Therefore, $211.2 \mathrm{~g}$ of the salt were dissolved in 11 of water as the molar weight is $422.4 \mathrm{~g} / \mathrm{mol}$. These simple experiments nevertheless demonstrate that a table-top beam line-like setup can nowadays be used in the lab to do ESCA type experiments. The two selected model systems also show the (well-known) element specificity as well as surface/interface sensitivity of the technique.

\subsubsection{Polarization-dependent measurements}

Polarization-dependent ESCA and XPS was for a long time only a domain of beam lines at large synchrotron facilities. The emission of photoelectrons from a sample is characteristic and displays an angular distribution [43]. It can be described in analogy to atomic spectroscopy [44]. Here we

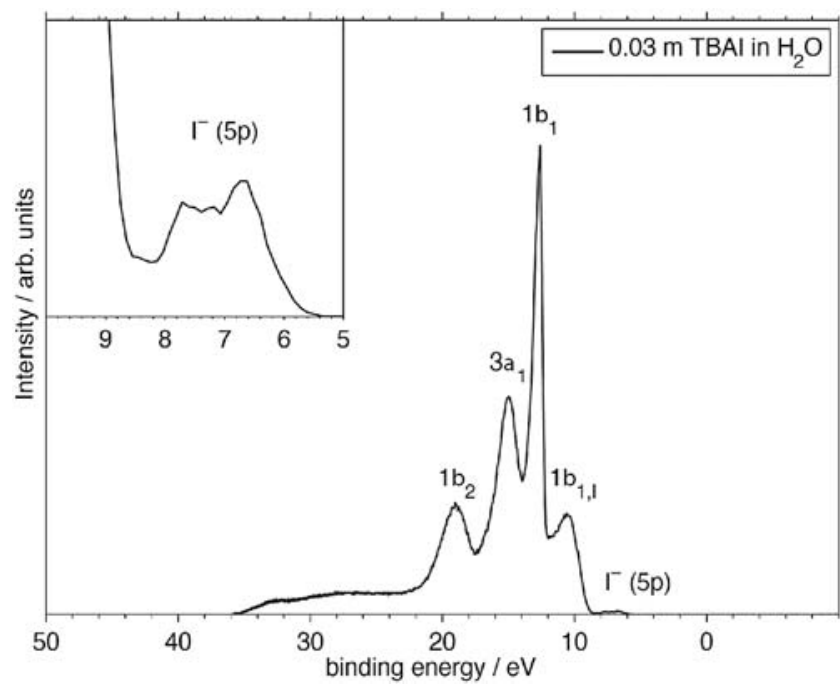

Fig. 10 Photoelectron spectra of a 0.03 molar solution of TBAI (tetrabutyl ammonium iodide) in liquid water. The spectrum displays two important things: (1) the element specificity and (2) TBAI is a surface-active molecule. During the formation of the liquid beam in the nozzle the TBAI enriches in the interface region, above a concentration of $0.02 \mathrm{~mol} / \mathrm{l}$ it assembles a monolayer on top of the water beam. The iodide ion is pulled to the interface as well and can be detected much easier than in cases where it is distributed homogeneously in the bulk

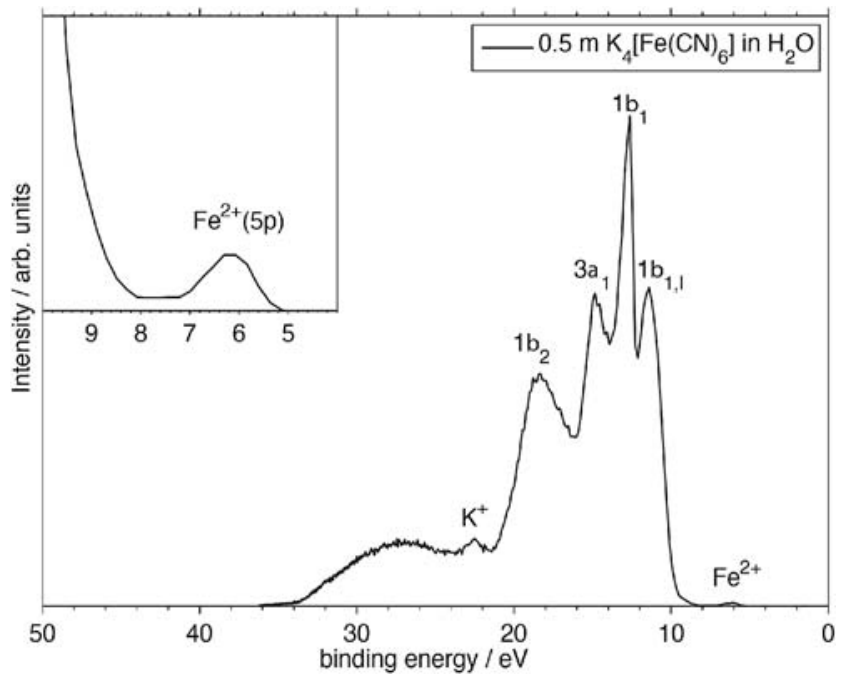

Fig. 11 Photoelectron spectra of a 0.5 molar aqueous solution of $\mathrm{K}_{4}\left[\mathrm{Fe}(\mathrm{CN})_{6}\right]$. The spectrum shows nicely the element specificity $\left(\mathrm{K}^{+}\right.$, $\mathrm{Fe}^{2+}$, water) of the liquid phase ESCA or XPS approach. The labels $1 b_{2}, 3 a_{1}, 1 b_{1}$, and $1 b_{1,1}$ mark the orbitals of gas and liquid phase water. The inset shows the Fe(II) line enlarged, demonstrating a good dynamic range and a decent signal-to-noise ratio

want to show that with laser driven high-harmonic tabletop setups these angular distributions can be determined by polarization-dependent measurements, because the polarization of the radiation in the experiments is well defined and can be controlled via the polarization of the fundamental laser radiation. 


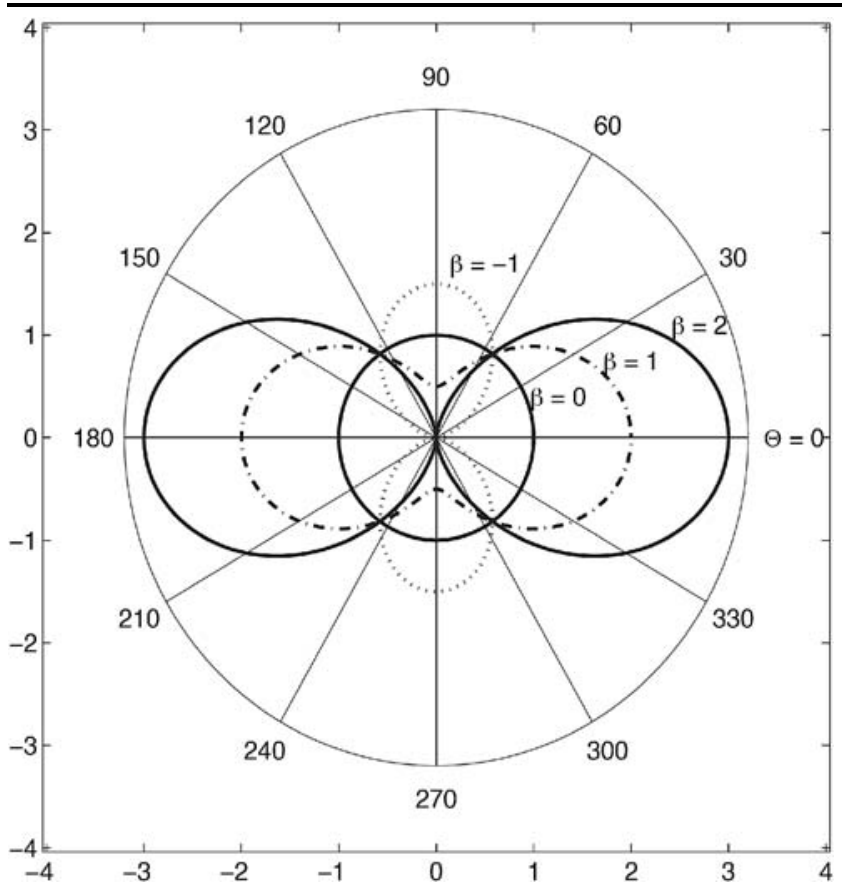

Fig. 12 Differential photoemission cross section $\mathrm{d} \omega / \mathrm{d} \Omega$ for different $\beta_{i}$-values but identical $\sigma_{I}$-values. The light is assumed to be horizontally polarized $\left(S_{1}=1\right)$

In order to fulfill the conservation of angular momentum, the change in momentum of the quantum system (atom, molecule) must be compensated for by the spatial distribution of photoelectrons. An electron, emitted from a $s$-orbital (quantum number $l=0$ ) with isotropic shape, has a spatial probability of emission which equals a dumbbell-shaped $p$-orbital (selection rule $\Delta l= \pm 1$ ). The emission from a $p$-orbital will on the other hand show a spatial distribution, which is a superposition of $s$ - and $d$-orbital-like shapes. Employing the dipole approximation the expression for the differential photoionization cross section of randomly oriented molecules in the direction $\Theta$ is $[43,45]$

$\frac{\mathrm{d} \sigma}{\mathrm{d} \Omega}=\left(\sigma_{i} / 4 \pi\right)\left[1+\left(\beta_{i} / 4\right)\left(1+3 S_{1} \cos 2 \Theta\right)\right]$.

$\beta_{i}$ is the energy-dependent anisotropy parameter, which lies in between 2 and -1 , and $\sigma_{i}$ is the relative partial photoemission cross section. Both $\beta_{i}$ and $\sigma_{i}$ are specific for a certain orbital und depend on the photon energy of the ionizing radiation $[45,46]$. The polarization of the light is expressed in terms of the Stokes parameter $S_{1}$ and is assumed to be linear (polarization direction: horizontal $S_{1}=1$, vertical $S_{1}=-1$ ) [46]. The angle between the light polarization and the emission direction of the photoelectron is $\Theta$.

Figure 12 shows the differential photoemission cross section for different $\beta_{i}$ values but the same $\sigma$ value (curves intersect at the magic angle of $54.7^{\circ}$ ). For $\beta=0$ the photoemission is isotropic. Nevertheless, all valence orbitals of gas phase water exhibit $\beta$ values $>0$, resulting in an anisotropic photoelectron distribution for which a higher photoemission in the direction of light polarization $\left(\Theta=0^{\circ}\right)$ than in the perpendicular direction $\left(\Theta=90^{\circ}\right)$ is observed.

When the photoemission angle equals $54.7^{\circ}$ the expression $\left(1+3 S_{1} \cos 2 \Theta\right)$ is zero and the differential photoemission cross section becomes independent of $\beta$. Under this angle all anisotropy effects disappear and the determination of the intensity ratios reveals directly the ratios of the corresponding $\sigma$ values.

Every orbital of the irradiated sample (water) generates a characteristic photoelectron signal depending on the angle of detection with respect to the polarization of the ionizing radiation. The intensity of this signal is governed by the energy- (wavelength-) dependent parameters $\sigma$ and $\beta$. The ratio of two peaks representing different species in the spectrum can be translated into their relative concentrations if the photoemission parameters for either component are known. For gas phase water the $\sigma$ - and the $\beta$-parameters were measured for photon energies in the range of 30 to $140 \mathrm{eV}$ [45]. In Table 1 we show the parameter for gas phase water for selected photon energies and orbitals close to the photon energy employed in our experiment $(38.6 \mathrm{eV})$ from [45] .

For the parameters at $38.5 \mathrm{eV}$ the differential photoemission cross sections for all valence orbitals of gas phase water were calculated, and they are depicted in Fig. 13. The ratio between the photoemission intensity at $0^{\circ}$ and at $90^{\circ}$ reveals the $\beta$ value ( $\sigma$ cancels out, (7b)).

$$
\begin{aligned}
\frac{I_{90^{\circ}}}{I_{0^{\circ}}} & =\frac{\sigma / 4 \pi}{\sigma / 4 \pi} \cdot \frac{1+(\beta / 4)\left(1+3 \cos 2 \cdot 90^{\circ}\right)}{1+(\beta / 4)\left(1+3 \cos 2 \cdot 0^{\circ}\right)} \\
& =\frac{1+(\beta / 4)(1-3)}{1+(\beta / 4)(1+3)}=\frac{1-\beta / 2}{1+\beta} .
\end{aligned}
$$

The ratio of the $\sigma$ values can be measured by tuning the detector position (or the polarization respectively) to the magic angle of $54.7^{\circ}$ degrees, where the $\beta$ dependence vanishes.

The Ti:Sa-laser radiation of our setup is horizontally polarized but its polarization direction can easily be tuned continuously with a $\lambda / 2$ waveplate inserted in the beam path in front of the high harmonics generation chamber. As the polarization of the high harmonics equals the polarization of the fundamental laser radiation [47, 48] - with an even reduced ellipticity [49] — the polarization of the extreme ultraviolet radiation can easily be tuned by rotating the polarization of the fundamental laser radiation. For the TOF spectrometer being fixed in the horizontal plane of the experiment a horizontal polarization of the XUV radiation represents $0^{\circ}$ whereas a vertical polarization equals a deviation angle of $90^{\circ}$ between the electrical light field and the detector axis.

Figure 14 shows a water spectrum (gas and liquid phase) at perpendicular polarizations of $0^{\circ}$ and $90^{\circ}$. The spectrum at $90^{\circ}$ displays a significantly reduced total intensity with regard to the $0^{\circ}$ spectrum (note: the intensity of the $90^{\circ}$ spec- 
Table 1 Tabulated $\beta$ and $\sigma$ values for orbitals of gas phase water. Values from [45]

\begin{tabular}{|c|c|c|c|c|c|c|}
\hline \multirow{3}{*}{$\begin{array}{l}\text { Molecular orbital } \\
\mathrm{MO}_{i}\end{array}$} & \multicolumn{3}{|c|}{$\beta_{i}$ (gas phase) } & \multicolumn{3}{|c|}{$\sigma_{i}($ gas phase $)$} \\
\hline & $E_{h v}$ & $E_{h v}$ & $\overline{E_{h v}}$ & $\overline{E_{h v}}$ & $E_{h v}$ & $E_{h v}$ \\
\hline & $36.5 \mathrm{eV}$ & $38.5 \mathrm{eV}$ & $42.7 \mathrm{eV}$ & $36.5 \mathrm{eV}$ & $38.5 \mathrm{eV}$ & $42.7 \mathrm{eV}$ \\
\hline $1 b_{1}$ & $1.39(5)$ & $1.38(5)$ & $1.48(3)$ & 1.00 & 1.00 & 1.00 \\
\hline $3 \mathrm{a}_{1}$ & $1.09(4)$ & $1.12(4)$ & $1.21(3)$ & $0.90(1)$ & $0.88(1)$ & $0.88(1)$ \\
\hline $1 b_{2}$ & $0.67(2)$ & $0.71(3)$ & $0.80(2)$ & $0.99(3)$ & $0.94(3)$ & $0.89(3)$ \\
\hline $2 \mathrm{a}_{1}$ & - & - & $1.27(7)$ & - & - & $0.31(3)$ \\
\hline
\end{tabular}

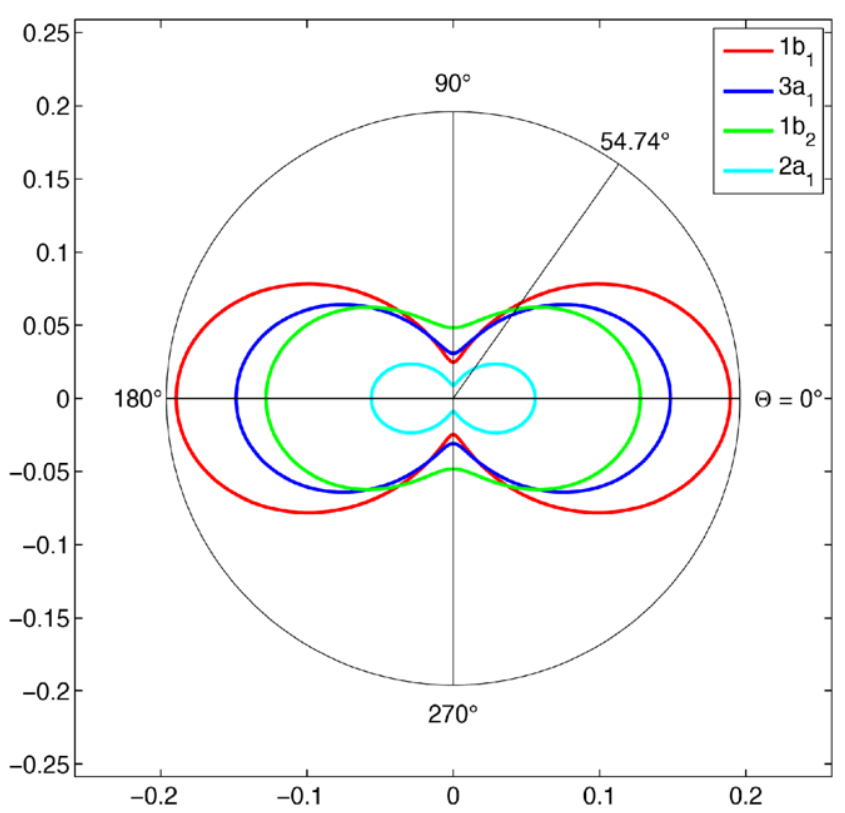

Fig. 13 For the parameters at $38.5 \mathrm{eV}$ the relative differential photoemission cross sections for all valence orbitals of gas phase water were calculated

trum is multiplied by a factor of three), which is in good overall agreement with theory and Figs. 12, 13 for $\beta$ values $>0$. Considering the transition from 0 to 90 degrees the ratio between the $1 b_{1, l i q}$ peak from the liquid and the $1 b_{1}$ peak from the gas phase is strongly increasing, showing a growing photoionization probability for the liquid phase with respect to the gas phase. This is mathematically represented by a smaller $\beta$ value for the liquid as a decreasing $\beta$ value leads to a more uniform (isotropic) emission and therefore reduces the intensity decline between $0^{\circ}$ and $90^{\circ}$. For a quantitative determination of the $\beta$ value the relevant peaks in the spectrum have been fitted with Gaussian curves. A comparison of the underlying areas results in a $I\left(90^{\circ}\right) / I\left(0^{\circ}\right)$ ratio of $1 / 3.73$ which is characteristic for an anisotropy parameter of $\beta=0.95$ in the liquid phase as opposed to a value of 1.38 in the gas phase. This value has not been measured before. The determination of the $\beta$ value for the $1 b_{1}$ orbital of liquid water shows the general power of this method to reveal photoemission parameters of single orbitals of liquids-in principle. However, the complex

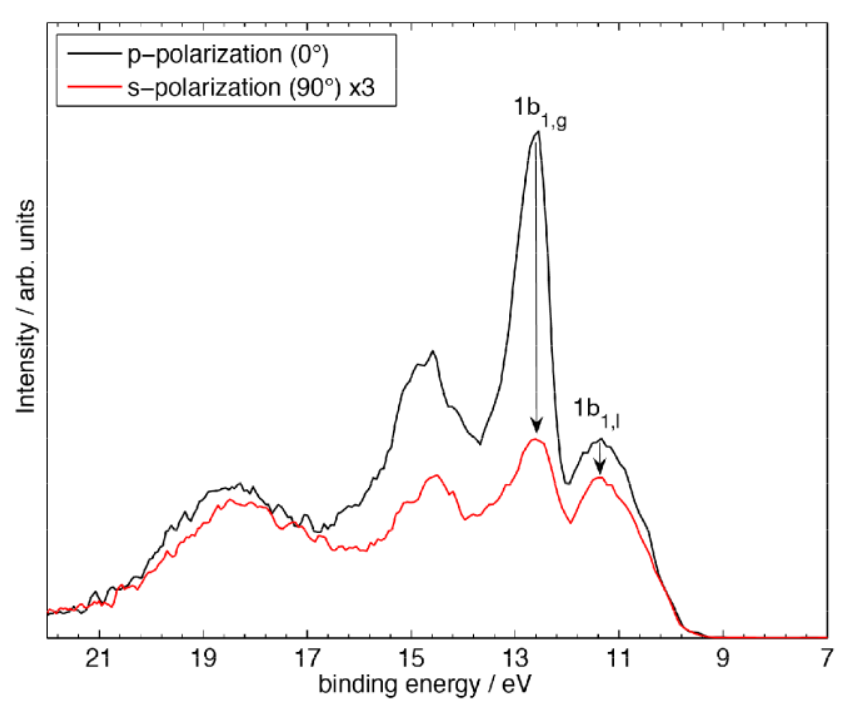

Fig. 14 Water spectrum (gas and liquid phase) at perpendicular polarizations of $0^{\circ}$ and $90^{\circ}$. The spectrum at $90^{\circ}$ displays a significantly reduced total intensity with regard to the $0^{\circ}$ spectrum (be aware that the intensity of the $90^{\circ}$ spectrum is multiplied by a factor of three), which is in perfect agreement with theory and Figs. 12, 13 for $\beta$ values $>0$

and overlapping nature of the photoelectron spectra and the so far limited energy resolution of the experimental setup make it difficult in this particular case to determine all $\sigma$ and $\beta$ parameters for all orbitals with high and sufficient accuracy. Also it appeared to be difficult to compare intensities at very large and very low binding energies quantitatively. For the ${ }^{1} b_{1}$ orbital of water in the gas and liquid phase, however, appearing in close proximity on the binding energy axis this was indeed easily possible. Therefore, the present approach is meant to show that these quantities may be determined in these experiments, in principle, and that they have to be measured, since they are different in the liquid and gas phase. While $\sigma$ and $\beta$ parameters of gas phase water are known and summarized in Table 1, those of the liquid phase are yet unknown.

\subsection{Dynamics in extreme states of water}

The dynamical structure of ambient water and the molecular picture of its important role in chemical and biological systems, often associated with a distinctive local struc- 
ture of hydrogen bonds $[9,50]$ has been studied with powerful spectroscopic techniques in the past [51-54]. Nevertheless, the degree of understanding is often not sufficient. Water at extreme temperature and pressure conditions is surprisingly also a field of continuous strong interest [55-57]. Its hydrogen bond structure has been investigated theoretically and experimentally [58]. Supercritical (sc) water plays an important role in many areas ranging from high temperature chemistry [59], hazardous waste treatment, technical processes and maybe the origin of life [60]. The dynamics of water and solutes have been studied mostly in static cells with spectroscopic techniques in the time [61] and frequency domain [59].

Recently we have reported on first investigations of the molecular photoelectron emission signature of the evolution of the phase and the hydrogen bonding network of superheated water below and above the critical point, as well as its time scales [1]. Here we report on a similar series of experiments, but for somewhat different experimental conditions. Also in this case we used an ultrafast femtosecond near IR laser pulse $(2.5-3 \mu \mathrm{m})$ to excite water resonantly via its $\mathrm{OH}$-stretch vibration inducing subsequent ultrafast heating to large internal energies and temperatures. The evolution of the superheated metastable water jet is again probed with pulsed ultrafast photoelectron emission spectroscopy. It is important to note that we observe approximately the same near interface molecules being initially in the first three monolayers [35] of the liquid and then in the dense evolving gas phase, throughout the evaporation process (for details see [1]). A time-series of spectra with perpendicular polarization of pump and probe beams is displayed in Fig. 15. As visible for negative delay times the EUV probe beam alone in the experiment ionizes water and produces photoelectrons from the ${ }^{1} b_{1},{ }^{3} a_{1},{ }^{1} b_{2}$, and ${ }^{2} a_{1}$ valence electrons of liquid water and some residual gas phase water $[5,8]$. The photoelectron spectrum of water is well known and prominent features for the valence electrons are easily assignable to the ${ }^{1} b_{1},{ }^{3} a_{1},{ }^{1} b_{2}$ orbitals (for assignments and features of orbitals of water see Fig. 16) of liquid and gas phase water [5, 8]. Similar to those in [1] the present spectra consist of superpositions of lines from the liquid and the gas phase of water. Narrower photoemission lines of the ${ }^{1} b_{1},{ }^{3} a_{1},{ }^{1} b_{2}$ electrons in the gas phase are overlapped with shifted (towards lower energy) and broad ${ }^{1} \mathrm{~b}_{1, \text { liq. }},{ }^{3} \mathrm{a}_{1, \text { liq. }}$, and ${ }^{1} \mathrm{~b}_{2, \text { liq }}$ lines of liquid water. The ${ }^{1} b_{1 \text {,liq. }}$ photoemission line can easily be identified, because it is the line with a large liquid-gas shift and observable in a region of least overlap at $11.16 \mathrm{eV}$ [5, 62]. As in [1] the time-resolved spectra in Fig. 15 have been plotted with a color code displaying the differences to the reference spectrum at negative delays, i.e., red displays a decrease and blue an increase in intensity relative to the reference spectrum. All spectra are averaged, normalized, and processed as described in detail in [1]. The Fig. 17 displays cuts through the spectra at a particular chemical shift (i.e., binding energy). In this case valuable kinetic traces can be obtained.

The interesting positions (chemical shift or binding energies) in the spectrum are the low-energy wing of the (static) liquid ${ }^{1} \mathrm{~b}_{1, \text { liq. }}$ photoemission line at $10.5 \mathrm{eV}$, its maximum at $11.16 \mathrm{eV}$ [62], a position between the ${ }^{1} b_{1}$ and the ${ }^{1} b_{1 \text {,iq. }}$ emission maximum at $11.6 \mathrm{eV}$, and the ${ }^{1} b_{1}$ gas phase emission maximum at $12.6 \mathrm{eV}$. While the $10.5 \mathrm{eV}$ and the $11.16 \mathrm{eV}$ energy windows monitor the decay of the liquid phase, the $11.6 \mathrm{eV}$ energy window monitors (although not exclusively) the appearance of the supercritical phase at short delays and the presence of clusters and water aggregates at later times. The chemical shift at $12.6 \mathrm{eV}$ monitors the increase of gas phase water at short delays. As we watch the spectra as a function of time (e.g., in Fig. 15), an initial fast band shift towards higher binding energy and a decay of the liquid ${ }^{1} \mathrm{~b}_{1, \text { liq. }}$. peak at $11.16 \mathrm{eV}$ is obvious (red). At the same time (for short delays) an increase of the ${ }^{1} b_{1}$ gas phase peak at 12.6 occurs (blue). Beyond these changes also intensity changes beyond $12.6 \mathrm{eV}$ occur which belong to contributions and intensities of photoemission peaks of the ${ }^{3} \mathrm{a}_{1},{ }^{1} \mathrm{~b}_{2}$ orbitals in liquid and gas phase water. For binding energies between 10.5 and $12.6 \mathrm{eV}$ the dynamics can be well followed in the time-resolved traces in Fig. 17.

As in [1] the internal energies of the excited water phase have been estimated from the known absorption coefficients of water [57] and determined to be $\approx 10$ and $\approx 40 \mathrm{~kJ} / \mathrm{mol}$ corresponding to transient temperatures of $\approx 400$ and $\approx 800 \mathrm{~K}$, respectively. While the first traces of Fig. 15 (left panel) correspond to temperatures of $400 \mathrm{~K}$ (strongly heated but well below the critical temperature $T_{\text {crit. }}$ ) the right panel displays experiments for $800 \mathrm{~K}$ corresponding to a supercritical fluid.

Beyond the overall features discussed above a new feature at later delay times is transient intensity in a range of the chemical shift (i.e., at $11.6 \mathrm{eV}$ ) between the liquid ${ }^{1} \mathrm{~b}_{1}$ peak at $11.16 \mathrm{eV}$ and the gas phase peak at $12.6 \mathrm{eV}$. It is known that in this energy range photoemission of clusters occurs $\left({ }^{1} b_{1}\right)[63,64]$. These features and trends are both observed in time-resolved spectra in Fig. 15 and the timeresolved traces in Fig. 17. We note here that the kinetics for the stronger excitation is faster in general.

As in a recent study we observe the evolution of a hot sub-critical and a supercritical phase with a characteristic spectral signature, being slightly different from that in [1]. The early time evolution in both experiments in Fig. 15 may be explained by a weakening of the hydrogen bonding network. Both experiments differ in the energy content of the hot water phase and the dynamics, which is characteristic of (i) fast evaporation and (ii) dispersion of the liquid. In the supercritical case the liquid phase photoemission vanishes 

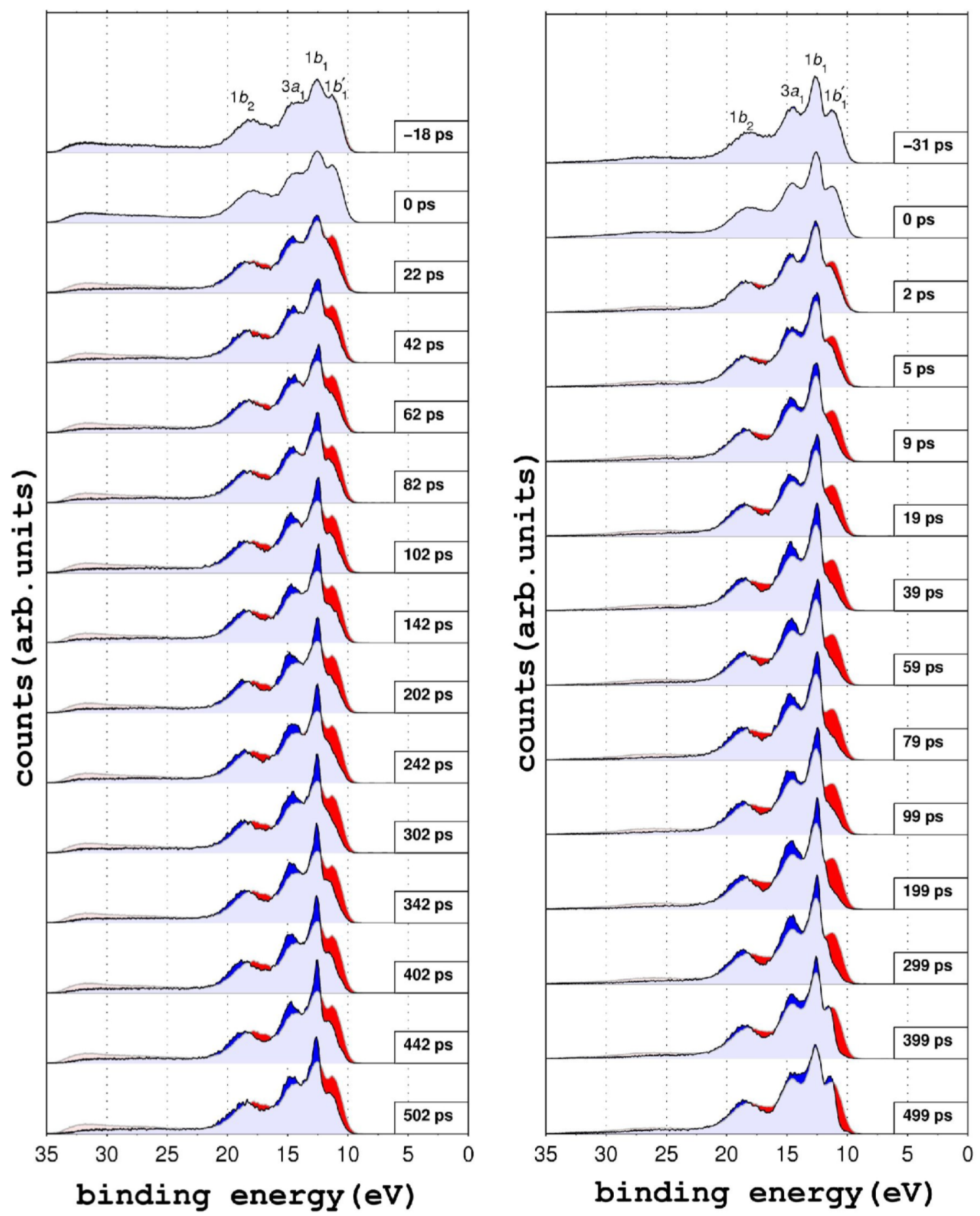

Fig. 15 Time-resolved photoelectron spectra of liquid (before excitation at negative delay time) and metastable water (left: excitation at $\lambda_{\mathrm{IR}}=2650 \mathrm{~nm}$; right panel: $\lambda_{\mathrm{IR}}=2830 \mathrm{~nm}$ :). For further details see the text

and the gas phase builds up temporarily. On a time scale of a few hundred picoseconds the gas phase vanishes again and photoemission in the $11.6 \mathrm{eV}$ energy range monitors more or less large clusters [63] either formed from the disintegrating liquid or through re-condensation of the gas phase or both, when aggregates from the disintegrating liquid serve as nu- cleation seeds. The disappearance of the gas phase may suggest the latter scenario at later times (Fig. 17). The present experimental results and those of [1] are obtained by gradually changing the energy content of the water through changing the excitation wavelength of the IR laser from 2650 to $2900 \mathrm{~nm}$ (maximum of absorption) or through changes of 
Fig. 16 Water and atom orbital energy levels (left) and plots of water orbitals at their binding energies (right)
Fig. 17 Kinetic traces at specific chemical shifts from time-resolved photoelectron spectra of water at 10.5, 11.16, 11.6 and $12.6 \mathrm{eV}$. Shown are kinetic traces for experiments with different IR pump wavelengths (2650 and $2830 \mathrm{~nm}$, respectively) and internal energies. For more details see the text
$\mathrm{H}_{2} \mathrm{O}$ energy levels
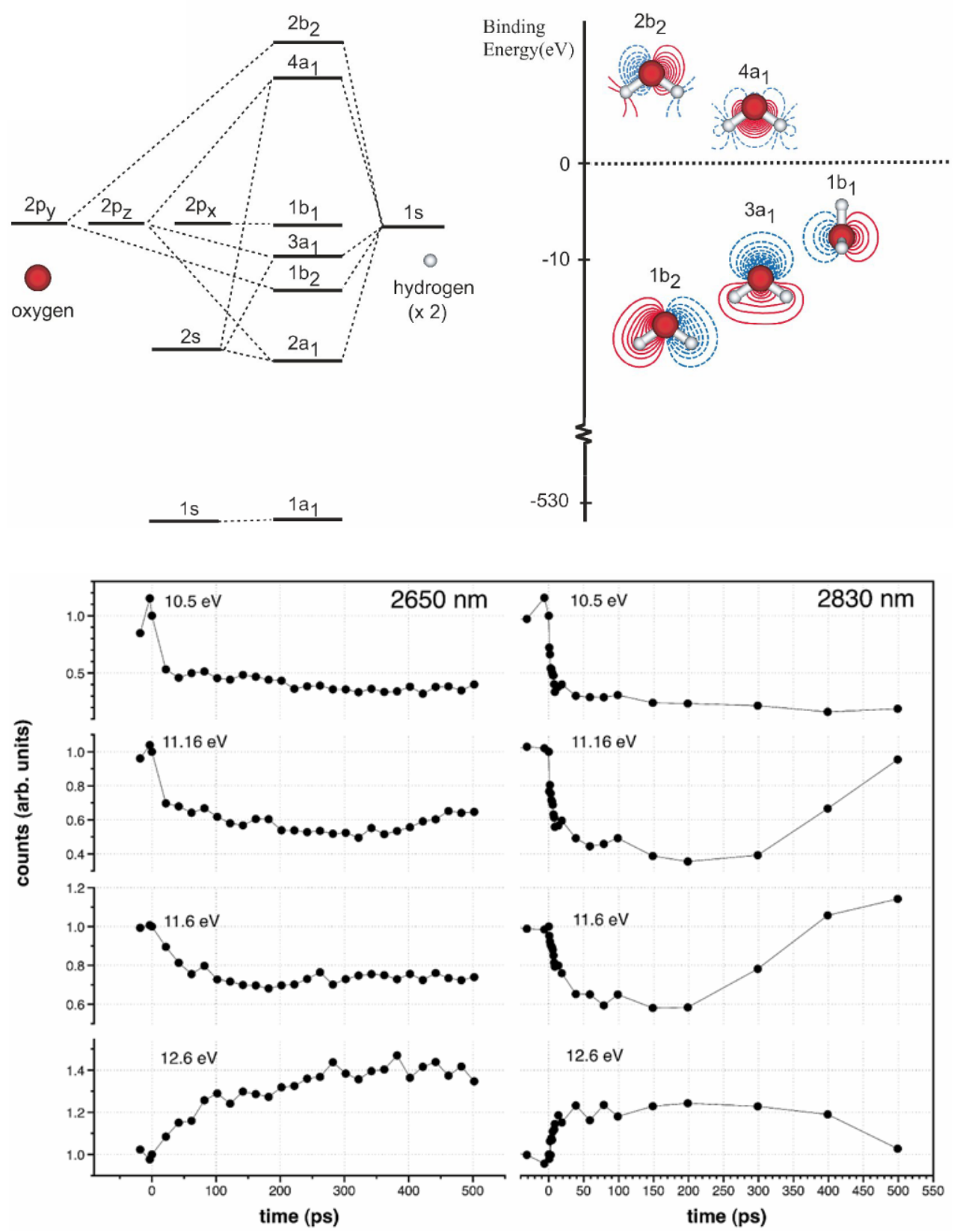

the pulse energy. In order to obtain more insight into the processes the time-resolved data have been compared with molecular dynamics simulations of the process $[1,65]$.

The Fig. 18 compares the situation for liquid ethanol which is excited at the maximum of its absorption band at $3 \mu \mathrm{m}$ and it is heated to nearly supercritical conditions. The assignments are taken from [8]. The difference to the water case is that the kinetics is significantly slower than in the case of superheated water. This phenomenon has been investigated in detail and compared with theory for methanol in [65]. Extensions of this technique to more chemical prob- lems (like the solvated electron in water) near liquid water interfaces is reported in [66].

\subsection{Dynamics of a laser induced plasma near a liquid surface}

Laser-ionized plasmas are of great interest owing to their unique properties and the fact that they suit many applications, such as the study of nuclear fusion, generation of energetic electrons and ions, $\mathrm{X}$-ray emission, $\mathrm{X}$-ray lasers and extreme-UV attosecond pulse generation (see [67] and refer- 


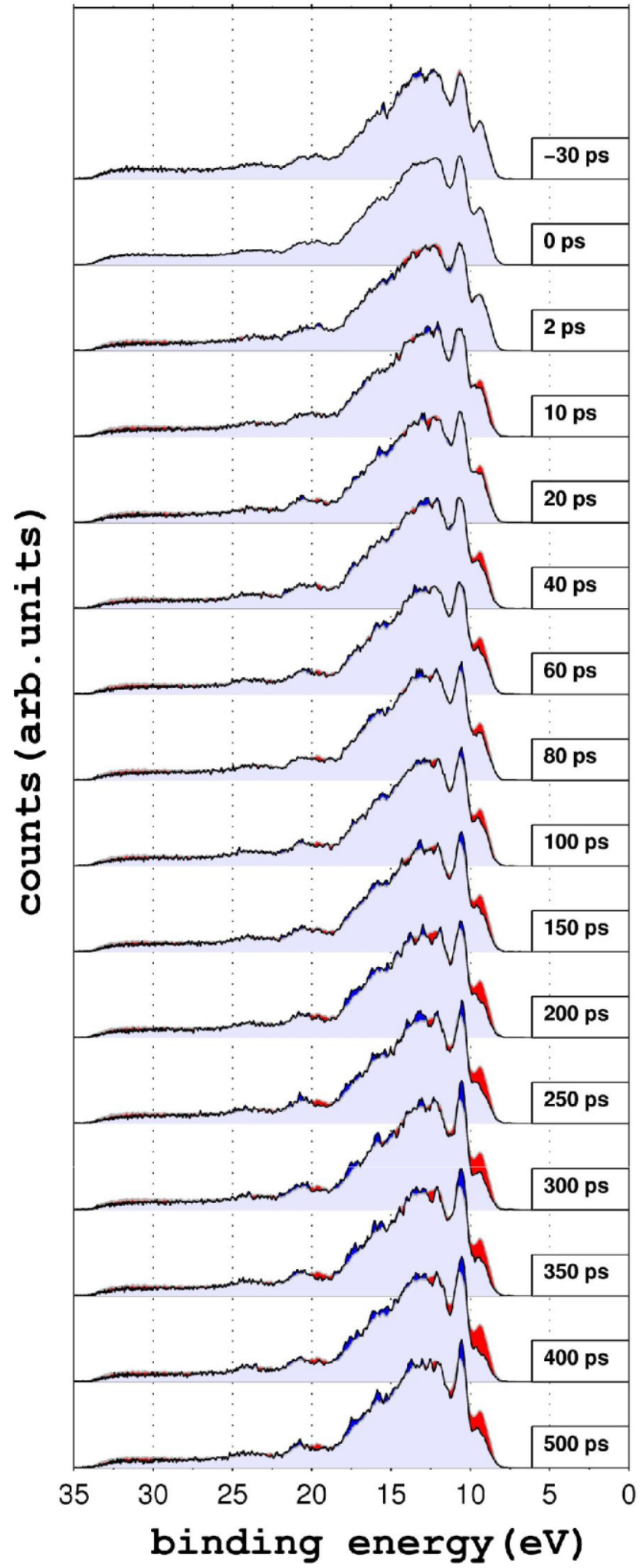

Fig. 18 Time-resolved photoelectron spectra of liquid (before excitation at neg. delays) and metastable ethanol (excited at $2950 \mathrm{~nm}$ : right). The photoemission line at the lowest binding energy corresponds to the liquid $3 \mathrm{a}^{\prime \prime}$ liq orbital of ethanol [8]. For further details see the text and [8] for assignments ences therein). A detailed knowledge of the plasma dynamics can be critical for optimizing a given application.

If the peak intensities of a short laser pulse reaches intensities around $10^{10} \mathrm{~W} / \mathrm{cm}^{2}$, ionization of atoms and molecules takes place by the absorption of multiple photons (multi photon ionization). At higher electrical field strength the mechanism of ionization may change via tunnel ionization towards field ionization $\left(>10^{14} \mathrm{~W} / \mathrm{cm}^{2}\right)$ [68]. These ionization processes are the initiating events, which in the end can lead to a plasma, if further in-coupling of laser energy in the medium occurs. In other words, a plasma is only formed if a certain free electron density is obtained which corresponds to a Debye length substantially smaller than the size of the system [69]. The heating of the plasma can occur via acceleration of charged particles in the oscillating electrical field $\left(10^{14}-10^{17} \mathrm{~W} / \mathrm{cm}^{2}\right)$ or via resonant excitation of collective electron motions (above $10^{17} \mathrm{~W} / \mathrm{cm}^{2}$ ). The second process needs a sufficiently high electron density so that the plasma oscillation can be in resonance with the laser frequency. For a vacuum wavelength of $800 \mathrm{~nm}$ the frequency is $375 \mathrm{THz}$, which corresponds to a free electron density of $1.7 \times 10^{21} \mathrm{~cm}^{-3}$.

The plasma oscillation was first described by Langmuir in the late 1920s [70]. A displacement of the electrons from their equilibrium position leads due to the restoring coulomb forces to collective electron motions, while the ions can approximately be considered to be motionless. The frequency of the electron oscillation is the well-known plasma frequency, which can be calculated as a function of the electron density $n_{e}$

$f_{e}=\frac{1}{2 \pi} \sqrt{\frac{e^{2} n_{e}}{\varepsilon_{0} m_{e}}}$

(in the absence of a magnetic field [71]).

Therefore, the plasma frequency provides information about the electron density and the degree of ionization of the plasma. The length scale, which divides individual particle behavior and collective behavior, is the Debye screening length $\lambda_{D}$. It is the distance over which an electric field is screened by redistribution of electrons in the plasma, and is given by [69]

$\lambda_{D}=\sqrt{\frac{\varepsilon_{0} k_{B} T}{e^{2} n}}$.

An ionized gas is not plasma, unless the Debye length is smaller than the size of the system.

Hot plasma produced by a femtosecond laser pulse does not start to expand immediately. The expansion velocity in a hot plasma may be as high as $30 \mu \mathrm{m} / \mathrm{ns}$ and for a cold neutral plasma expansion velocities of about $100 \mathrm{~m} / \mathrm{s}$ were found at electron temperatures around $1000 \mathrm{~K}$ [71]. The expansion was monitored by the temporal evolution of the plasma 
frequency, which was probed by a resonant RF probe. The ponderomotive force leads to a displacement of electrons in the direction of pulse propagation [72]. As a plasma oscillation is induced by such charge displacements it is expectable that a longitudinal electron (plasma) oscillation in the same direction as the light propagation is emerging. In terms of macroscopic charging an oscillating dipole moment on the optical axis is generated which in turn can emit electromagnetic radiation perpendicular to this axis. Such fast pulsed electric fields were experimentally found in selfgenerated filaments in air after excitation by a femtosecond laser pulse [73].

Various methods have been applied to investigate the parameters of laser-induced plasmas. The electron temperature has been measured by Thomson scattering [74]. The ionization stage has been determined by ion spectrometry [75] and by recording the emission of X-rays from the plasma [76]. The time-resolved plasma density profile has been measured by optical interferometry and holography [77, 78]. Moiré deflectometry has been used to determine the density profile in the plasma channel and its lateral expansion [79]. Spectrometry of ions emitted from the plasma has yielded information on ion velocity and temperature [80].

Using optical near IR fs-pulses and high-harmonic radiation we have investigated the dynamics, i.e., the oscillation and decay of a plasma near a liquid water jet as well as some of its characteristic parameters.

The presented results were recorded with the IR-pumpXUV-probe apparatus described above. At the IR wavelength of $2650 \mathrm{~nm}$, which was used for exciting the plasma, typically a pulse energy of $20-30 \mu \mathrm{J}$ was attained for the nonlinear photon conversion process. This is substantially higher than for the experiments described in Sec. 3.2, and also the radiation was tightly focused. Under the assumption of a pulse duration of $200 \mathrm{fs}$ [36] and a focus diameter of $80-100 \mu \mathrm{m}$ still a peak intensity of well above $10^{12} \mathrm{~W} / \mathrm{cm}^{2}$ is reached. For the probe the 25th harmonic of $800 \mathrm{~nm}$ radiation, corresponding $38.6 \mathrm{eV}$ photon energy, was chosen. In the vicinity of a liquid beam in vacuum a radial power low decay of the gas phase density from the liquid surface toward the vacuum was documented [5].

In Fig. 19 time-resolved kinetic energies (upper trace) and apparent "binding energies" (lower trace) of an oscillating near liquid interface plasma as a function of time displaying distinct oscillations are depicted. We provide both the kinetic energy trace and the corresponding (theoretically calculated) binding energy. The oscillating "binding energy" suggests that the electrons stem from and belong to the water molecules, however, they are not bound anymore to the water in the gas phase but both are part of an ensemble of ionized cations and electrons oscillating in time. The plot in energetic scale shows photoelectrons with large kinetic energies close to the photon energy.
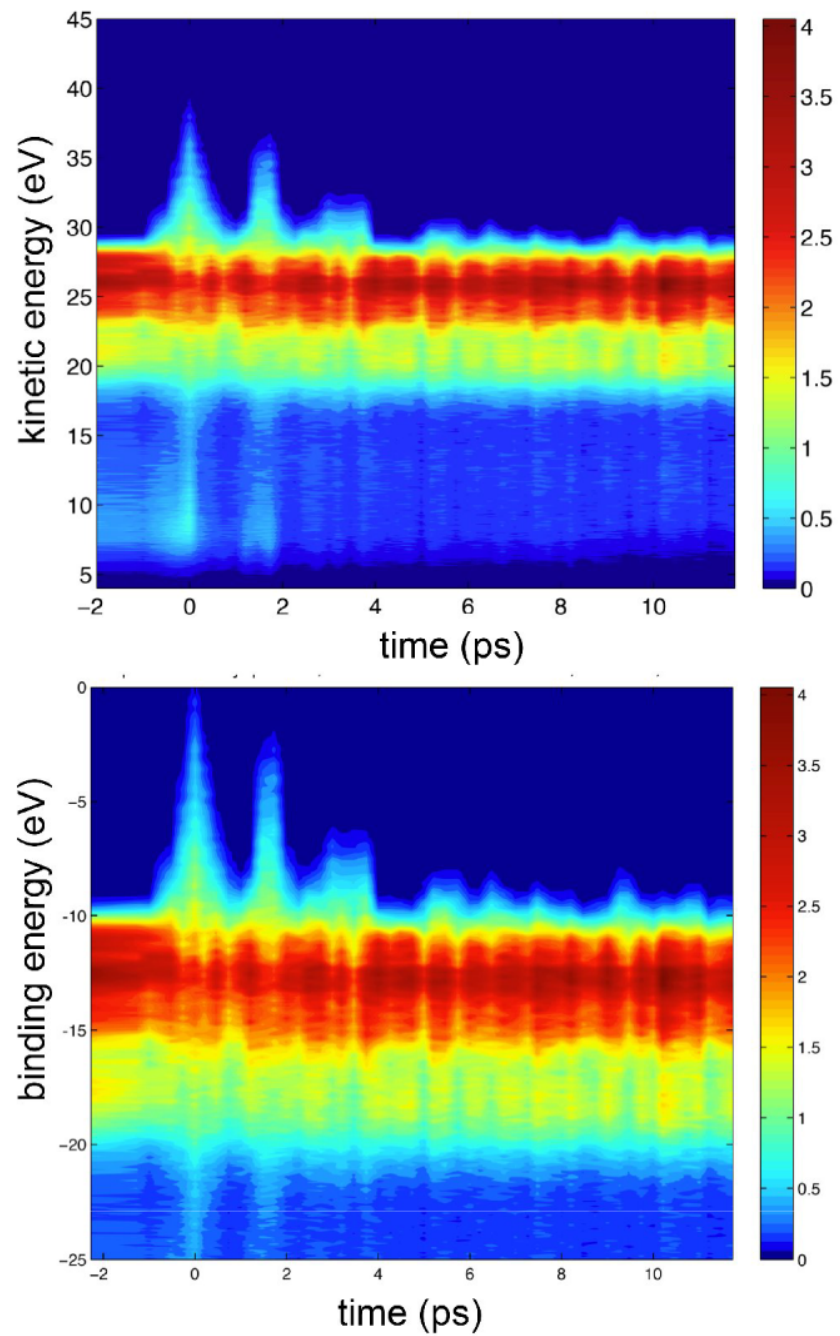

Fig. 19 Kinetic energies of photoelectrons as a function of time on top of the water photoelectron spectrum (vertical cut) in the case of an oscillating near liquid water surface plasma. An oscillation period of the fast electrons of $1.5 \mathrm{ps}$ corresponding to a plasma frequency of $0.67 \mathrm{THz}$ and a free electron density of $5.6 \times 10^{15} \mathrm{~cm}^{-3}$ (for more details see the text)

At time delay 0 the IR pump pulse initiates plasma formation near the liquid beam interface (Fig. 20a). According to (8) a period of $1.5 \mathrm{ps}$ corresponds to a plasma frequency of $0.67 \mathrm{THz}$ and a free electron density of $5.6 \times$ $10^{15} \mathrm{~cm}^{-3}$. This is a quite low level of electron density. At $2650 \mathrm{~nm}$ the resonance frequency of a laser driven plasma is $113 \mathrm{THz}$, which corresponds to a free electron density of $1.6 \times 10^{20} \mathrm{~cm}^{-3}$. This number is more than one order of magnitude lower than the critical resonance free electron density for $800 \mathrm{~nm}$ radiation. Due to the low free electron density in the plasma we conclude that the plasma resembles more a cold plasma ionized via ponderomotive acceleration of electrons and tunnel ionization rather than normal plasma heating at the plasma frequency. Although a number of 22 photons at $2650 \mathrm{~nm}$ would be nec- 

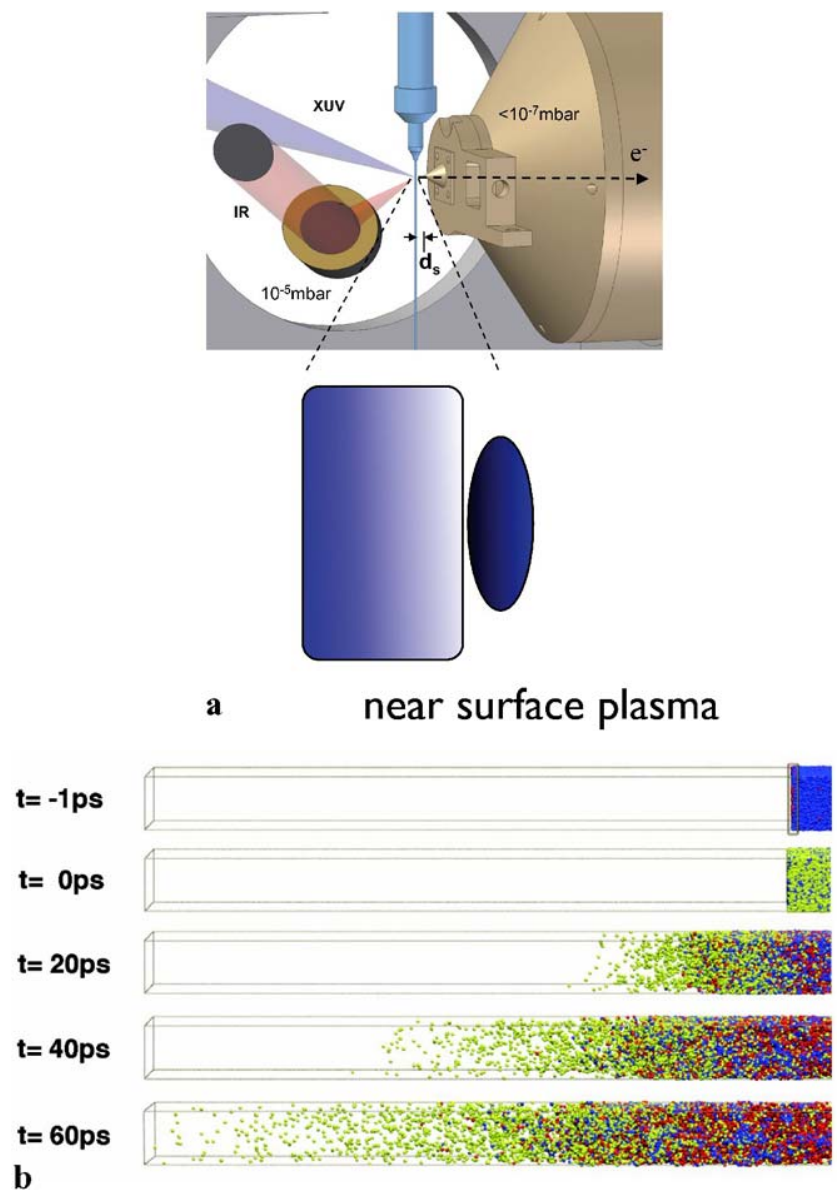

Fig. 20 (a) Laser-generated plasma near a liquid water jet (schematic). (b) Decomposing surface of a liquid interface as calculated in a molecular dynamics simulation. This time scale determines the decay of the oscillation (and actually terminates it) of the plasma on a picosecond time scale. The dimensions of the simulation boxes in (b) are $4 \mathrm{~nm} \times 4 \mathrm{~nm} \times 50 \mathrm{~nm}[1,65]$ with 300000 water molecules

essary to result in an ionization energy of $10 \mathrm{eV}$, making true multi-photon process less likely, together with the arguments above, some vibrational resonance contribution within the ionization process has been observed from the wavelength dependence of the process. The time scale of the decay of the plasma in the few picoseconds regime (see Fig. 19) is related to the transport of hot water molecules in the expanding (exploding) beam interface, which has been estimated from molecular dynamics simulations (Fig. 20b).

In conclusion, we demonstrated a method for real-time probing of laser-ionized plasmas with high temporal resolution, yielding information that is not accessible by other methods. The technique, based on IR pump and EUV probe, yields two-dimensional spectral information about a gas phase plasma expanding near a liquid water beam. As shown above, from the overall features the ionization mechanism may be inferred.

\section{Summary and conclusions}

In the present contribution we have provided an overview of the technical details and some specific applications of ultrafast electronic spectroscopy for chemical analysis (ESCA) near liquid interfaces (being conceptually an ultrafast extreme ultraviolet photoelectron spectroscopy). We have shown that this is a powerful and novel two-dimensional spectroscopic tool that can monitor (valence) electron orbital energies and chemical shifts in time in the condensed phase near liquid interfaces, and that it enables a new avenue for monitoring ultrafast processes in chemistry and in biological model systems near water and other liquid solvents interfaces. In a few applications of the technique, we have shown the advantage of the static table-top approach (photoelectron spectra near liquid interfaces have not been recorded with high-harmonic sources before) and we have shed light onto the dynamics of extreme states of matter (e.g. superheated and supercritical water and ethanol) and a surface plasma which are all interesting both from a fundamental point of view and for certain analytical applications.

The current state-of-the art report of our technology (to our knowledge, this is the only experiment of this kind to date) demonstrates the high potential of the unique experiment. One of the main future goals of our group will be to develop the current technique into an ultrafast photoemission spectroscopy (in the spirit of liquid interface ESCA) accessing core electrons in the so-called water window at photon energies between 200 and $600 \mathrm{eV}$. The basic strategies for this have been worked out in the SPP1134 priority program of the DFG. Moreover, in the future we aim to trace molecular orbitals (and their autocorrelation functions) during molecular transformations near liquid interfaces, in close collaboration with theory. One of the main challenges will be the clear distinction of liquid surface phenomena and processes in the bulk (below the first mono layer), which could in principle be addressed in $\mathrm{E}(\mathrm{X}) \mathrm{UV}$ wavelength-dependent experiments (due to different probing and electron escape depths).

Acknowledgements This work was supported by the Deutsche Forschungsgemeinschaft (SPP1134, "Investigation of Transient Structures of Photo-Induced Chemical Reactions in Solution with Ultrafast XUV-Photoelectron Spectroscopy", Project AB63/7-1,2, SFB 755, "Nanoscale Photonic Imaging", Project B2, GK782, "Dynamics and spectroscopy of molecular aggregates, coils and networks"), the Fonds der Chemischen Industrie, as well as by the State of Lower Saxony and the Volkswagen Foundation.

\section{References}

1. O. Link, E. Vöhringer-Martinez, E. Lugovoy, Y. Liu, K. Siefermann, M. Faubel, M. Grubmüller, R.B. Gerber, Y. Miller, B. Abel, Faraday Discuss. 141, 67 (2009) 
2. K. Siegbahn, D. Hammond, H. Fellner-Feldegg, E.F. Barnett, Science 176(32), 245 (1972)

3. K. Siegbahn, Science 217, 111 (1982)

4. K. Siegbahn, H. Svensson, M.J. Lundholm, J. Electron Spectrosc. 24, 205 (1981)

5. M. Faubel, in Adv. Series in Physical Chemistry, vol. 10A, ed. by C.Y. $\mathrm{Ng}$ (World Scientific, New York, 2000)

6. M. Faubel, T. Kisters, Nature 339, 527 (1989)

7. M. Faubel, S. Schlemmer, J.P. Toennies, Z. Phys. D, At. Mol. Clust. 10, 269 (1988)

8. M. Faubel, B. Steiner, J.P. Toennies, J. Chem. Phys. 106, 9013 (1997)

9. B. Winter, E.F. Aziz, U. Hergenhahn, M. Faubel, I.V. Hertel, J. Chem. Phys. 126(12), 124504 (2007)

10. B. Winter, M. Faubel, Chem. Rev. 106(4), 1176 (2006)

11. U. Gelius, Phys. Scr. 9, 133 (1974)

12. X.F. Li, A. L'Huillier, M. Ferray, L.A. Lompre, G. Mainfray, Phys. Rev. A 39, 5751 (1989)

13. E. Seres, J. Seres, F. Krausz, C. Spielmann, Phys. Rev. Lett. 92, $163002(2004)$

14. P. Corkum, Phys. Rev. Lett. 71, 1994 (1993)

15. P. Jaegle, Coherent Sources of XUV Radiation (Springer, New York, 2006)

16. T.F. Gallager, Phys. Rev. Lett. 61, 2304 (1988)

17. M. Lewenstein, P. Balcou, M.Y. Ivanov, A. L'Huillier, P. Corkum, Phys. Rev. A 49, 2117 (1994)

18. L. Nugent-Glandorf, M. Scheer, D.A. Samuels, A.M. Mulhisen, E.R. Grant, X. Yang, V.M. Bierbaum, S.R. Leone, Phys. Rev. Lett. 87, $193002(2001)$

19. A. Paul, R.A. Bartels, R. Tobey, H. Green, S. Weiman, I.P. Christov, M.M. Murnane, H.C. Kapteyn, S. Backus, Nature 421(6918), $51(2003)$

20. E.A. Gibson, A. Paul, N. Wagner, R. Tobey, D. Gaudiosi, S. Backus, I.P. Christov, A. Aquila, E.M. Gullikson, D.T. Attwood, M.M. Murnane, H.C. Kapteyn, Science 302(5642), 95 (2003)

21. S. Kim, J. Jin, Y.-J. Kim, I.-Y. Park, Y. Kim, S.-W. Kim, Nature 453, 757 (2008)

22. R. Kienberger, E. Goulielmakis, M. Uiberacker, A. Baltuska, V. Yakovlev, F. Bammer, A. Scrinzi, T. Westerwalbesloh, U. Kleineberg, U. Heinzmann, M. Drescher, F. Krausz, Nature 427(6977), 817 (2004)

23. T. Morishita, S. Watanabe, C.D. Lin, Phys. Rev. Lett. 98(8), 083003 (2007)

24. M. Hentschel, R. Kienberger, C. Spielmann, G.A. Reider, N. Milosevic, T. Brabec, P. Corkum, U. Heinzmann, M. Drescher, F. Krausz, Nature 414(6863), 509 (2001)

25. M. Lein, Phys. Rev. Lett. 94(5), 053004 (2005)

26. H. Kapteyn, O. Cohen, I. Christov, M. Murnane, Science 317(5839), 775 (2007)

27. P. Johnsson, R. Lopez-Martens, S. Kazamias, J. Mauritsson, C. Valentin, T. Remetter, K. Varjú, M.B. Gaarde, Y. Mairesse, H. Wabnitz, P. Salières, P. Balcou, K.J. Schafer, A. L'Huillier, Phys. Rev. Lett. 95(1), 013001 (2005)

28. S.A. Aseyev, Y. Ni, L.J. Frasinski, H.G. Muller, M.J. Vrakking, Phys. Rev. Lett. 91(22), 223902 (2003)

29. A.L. Cavalieri, N. Möller, T. Uphues, V.S. Yakovlev, A. Baltuska, B. Horvath, B. Schmidt, L. Blümel, R. Holzwarth, S. Hendel, M. Drescher, U. Kleineberg, P.M. Echenique, R. Kienberger, F. Krausz, U. Heinzmann, Nature 449(7165), 1029 (2007)

30. H. Niikura, F. Lögar, R. Hasbani, A.D. Bandrauk, M.Y. Ivanov, D.M. Villeneuve, P.B. Corkum, Nature 417(6892), 917 (2002)

31. A. Staudte, D. Pavicić, S. Chelkowski, D. Zeidler, M. Meckel, H. Niikura, M. Schäffler, S. Schössler, B. Ulrich, P.P. Rajeev, T. Weber, T. Jahnke, D.M. Villeneuve, A.D. Bandrauk, C.L. Cocke, P.B. Corkum, R. Dörner, Phys. Rev. Lett. 98(7), 073003 (2007)
32. M. Drescher, M. Hentschel, R. Kienberger, M. Uiberacker, V. Yakovlev, A. Scrinzi, T. Westerwalbesloh, U. Kleineberg, U. Heinzmann, F. Krausz, Nature 419(6909), 803 (2002)

33. P. Agostini, L.F. DiMauro, Rep. Prog. Phys. 67, 813 (2004)

34. M. Drescher, Z. Phys. Chem. 218, 1147 (2004)

35. Y. Itikawa, N. Mason, J. Phys. Chem. Ref. Data 34, 1 (2005)

36. U. Emmerichs, S. Woutersen, H.J. Bakker, J. Opt. Soc. Am. 14, 1480 (1997)

37. J. Jasny, U. Teubner, W. Theobald, C. Wäulker, J. Bergmann, F.P. Schäfer, Rev. Sci. Instr. 65, 1631 (1994)

38. Y. Lim, T. Westerwalbesloh, A. Aschentrup, A. Wehmeyer, G. Haindl, U. Kleineberg, U. Heizmann, Appl. Phys. A 72, 121 (2001)

39. B. Winter, R. Weber, I.V. Hertel, M. Faubel, P. Jungwirth, E.C. Brown, S.E. Bradforth, J. Am. Chem. Soc. 127(19), 7203 (2005)

40. H. Siegbahn, K. Siegbahn, J. Electron Spectrosc. Relat. Phenom. 2, 319 (1973)

41. Q. Du, R. Superne, E. Freysz, Y.R. Shen, Phys. Rev. Lett. 70, 2313 (1993)

42. I.F.W. Kuo, C.J. Mundy, Science 303, 658 (2004)

43. J. Cooper, R.N. Zare, J. Chem. Phys. 48, 942 (1968)

44. J.H.D. Eland, Photoelectron Spectroscopy — an Introduction to Ultraviolet Photoelectron Spectroscopy in the Gas Phase (Butterworth, New York, 1974)

45. M.S. Banna, B.H. McQuaide, R. Malutzki, V. Schmidt, J. Chem. Phys. 84, 4739 (1986)

46. E. Hecht, Optik (Oldenbourg, New York, 2005)

47. P. Antoine, A. l'Huillier, S. Lewenstein, P. Salières, B. Carre, Phys. Rev. A 53, 1725 (1996)

48. D. Schulze, M. Dörr, G. Sommerer, J. Ludwig, P.V. Nickles, T. Schlegel, W. Sandner, M. Drrescher, U. Kleineberg, U. Heinzmann, Phys. Rev. A 57, 3003 (1998)

49. J.G. Eden, Prog. Quantum Electron. 28, 197 (2004)

50. P. Wernet, D. Nordlund, U. Bergmann, M. Cavalleri, M. Odelius, H. Ogasawara, L.A. Nyslund, T.K. Hirsch, L. Ojamße, P. Glatzel, L.G. Pettersson, A. Nilsson, Science 304(5673), 995 (2004)

51. Z. Wang, Y. Pang, D.D. Dlott, J. Phys. Chem. 111(17), 3196 (2007)

52. S. Ashihara, N. Huse, A. Espagne, E.T. Nibbering, T. Elsaesser, J. Phys. Chem. A 111(5), 743 (2007)

53. M.L. Cowan, B.D. Bruner, N. Huse, J.R. Dwyer, B. Chugh, E.T. Nibbering, T. Elsaesser, R.J. Miller, Nature 434(7030), 199 (2005)

54. S. Woutersen, H.J. Bakker, Phys. Rev. Lett. 96(13), 138305 (2006)

55. A.F. Goncharov, N. Goldmann, L.E. Fried, C. Crowhurst, I.-F. Kuo, W.C.J. Mundy, J.M. Zaug, Phys. Rev. Lett. 94, 125508 (2005)

56. F. Bencivenga, A. Cunsolo, M. Krisch, G. Monaco, G. Ruocco, F. Sette, Phys. Rev. E 75(5 Pt 1), 051202 (2007)

57. A. Charvat, B. Stasicki, B. Abel, J. Phys. Chem A 110(9), 3297 (2006)

58. N. Goldmann, L.E. Fried, J. Chem. Phys. 126, 134505 (2007)

59. N. Akiya, P.E. Savage, Chem. Rev. 102, 2725 (2002)

60. L.J. Rothschild, R.L. Mancinelli, Nature 409, 1092 (2001)

61. T.B. Brill, J. Phys. Chem. A 104, 4343 (2000)

62. H. Agren, V. Carravetta, Mol. Phys. 55, 901 (1985)

63. L. Belau, K.R. Wilson, S.R. Leone, M. Ahmed, J. Phys. Chem. A 111, 10075 (2007)

64. O. Björnholm, F. Federmann, S. Kakar, T. Moller, J. Chem. Phys. 111, 546 (1999)

65. O. Link, E. Vöhringer-Martinez, Y. Liu, K. Siefermann, E. Lugovoy, M. Faubel, H. Grubmüller, B. Abel, (2009, submitted)

66. K. Siefermann, Y. Liu, E. Lugovoy, O. Link, M. Faubel, U. Buck, B. Winter, B. Abel, (2009, submitted)

67. M. Centurion, P. Reckenthaler, S.A. Trushin, F. Krausz, E.E. Fill, Nat. Photonics 2, 315 (2008) 
68. H. Schwoerer, Femtosecond Technology for Technical and Medical Applications (Springer, Berlin, 2004)

69. T.C. Kilian, Phys. Rev. Lett. 83, 4776 (1999)

70. L. Tonks, I. Langmuir, Phys. Rev. A 33, 82 (1929)

71. S. Kulin, Phys. Rev. Lett. 85, 318 (2000)

72. J.H. Eberly, Phys. Rev. 176, 1570 (1968)

73. A. Proulx, Opt. Commun. 174, 305 (2000)

74. T.E. Glover, T.D. Donnelly, E.A. Lipman, A. Siullivan, R.W. Falcone, Phys. Rev. Lett. 73, 78 (1994)

75. S. August, D.D. Meyerhofer, D. Strickland, S.L. Chin, J. Opt. Soc. Am. B 8, 858 (1991)
76. B.E. Lemoff, G.Y. Yin, C.L. Gordon III, C.P.J. Barty, S.E. Harris, Phys. Rev. Lett. 74, 1574 (1995)

77. S. Tsortzakis, B. Prade, M. Franco, A. Mysyrowicz, Opt. Commun. 181, 123 (2000)

78. M. Centurion, Y. Pu, D. Psaltis, T.W. Häntsch, Opt. Lett. 29, 772 (2004)

79. M. Dunne, T. Afshar-Rad, J. Edwards, A.J. MacKinnon, S.M. Viana, O. Willi, G. Pert, Phys. Rev. Lett. 72, 1024 (1994)

80. L.J. Frasinski, K. Codling, P. Hatherly, J. Barr, I.N. Ross, W.T. Toner, Phys. Rev. Lett. 58, 2424 (1987) 